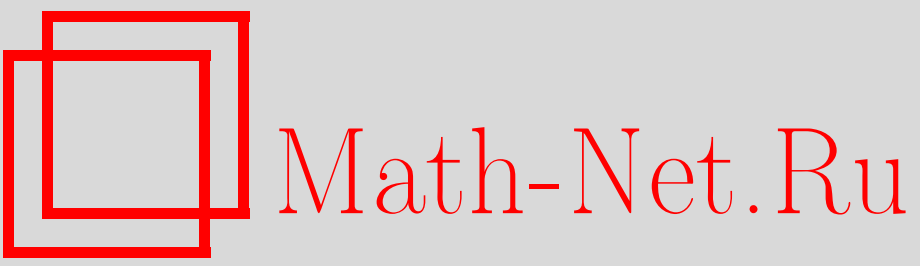

Б. Мампория, Стохастическое дифференциальное уравнение для обобщенных случайных процессов в банаховом пространстве, Теория вероятн. и ее примен., 2011, том 56, выпуск 4, 704-725

DOI: https://doi.org/10.4213/tvp4419

Использование Общероссийского математического портала Math-Net.Ru подразумевает, что вы прочитали и согласны с пользовательским соглашением

http://www. mathnet.ru/rus/agreement

Параметры загрузки:

IP: 54.198 .187 .58

26 апреля 2023 г., 15:27:17 
ТЕОРИЯ ВЕРОЯТНОСТЕЙ

Том 56

И ЕЕ ПРИМЕНЕНИЯ

Выпуск 4

2011

(c) 2011 г.

МАМПОРИЯ Б. И.*

\title{
СТОХАСТИЧЕСКОЕ ДИФФЕРЕНЦИАЛЬНОЕ УРАВНЕНИЕ ДЛЯ ОБОБЩЕННЫХ СЛУЧАЙНЫХ ПРОЦЕССОВ В БАНАХОВОМ ПРОСТРАНСТВЕ ${ }^{1)}$
}

\begin{abstract}
Определен стохастический интеграл от неупреждающих обобщенных случайных процессов (неупреждающих случайных процессов) по числовому винеровскому процессу в произвольном сепарабельном банаховом пространстве. Вопрос о существовании стохастического интеграла как случайного элемента в банаховом пространстве сведен к задаче о разложимости обобщенного случайного элемента. Рассмотрено стохастическое дифференциальное уравнение для обобщенного случайного процесса и изучен вопрос о существовании и единственности решения. Выводятся соответствующие результаты для стохастического дифференциального уравнения в банаховом пространстве.
\end{abstract}

Ключевые слова и фразы: стохастический интеграл Ито, стохастическое дифференциальное уравнение, винеровский процесс, ковариационные операторы в банаховом пространстве.

1. Введение. В конечномерном случае стохастический интеграл Ито определяется сперва для неупреждающих ступенчатых функций, а затем для процессов второго порядка как предел стохастических интегралов от неупреждающих ступенчатых функций, сходящихся к данной функции. Такой подход позволяет построить стохастические интегралы в банаховых пространствах со специальной геометрической структурой (см. [6], [8], [12]). Однако в произвольных банаховых пространствах можно таким путем построить стохастический интеграл только в том случае, если интегрируемая функция неслучайна (см. [9]). Аналогичная ситуация создается, когда интегрируемая функция операторнозначна и винеровский процесс принимает значения в банаховом пространстве (см. $[1],[3],[7])$. Наш подход к этой задаче таков: мы определяем стохастический интеграл для обобщенных случайных процессов (значит и для широкого класса случайных процессов), который является обобщенным

\footnotetext{
* Институт вычислительной математики им. Н.И. Мусхелишвили Грузинского технического университета, ул. Акурская 8, 0171 Тбилиси, Грузия; е-mail: badrimamporia@yahoo.com

1) Работа выполнена при поддержке гранта \#GNSF/STO9_99_3-104.
} 
случайным элементом; если он разложим, т.е. представляется случайным элементом, то мы говорим, что этот случайный элемент является стохастическим интегралом. Таким образом, вопрос существования стохастического интеграла сводится к хорошо известной задаче о представлении обобщенного случайного элемента случайным элементом. Далее мы рассматриваем стохастическое дифференциальное уравнение и исследуем вопрос существования и единственности решения в виде обобщенного случайного процесса. Полученный результат применяем для исследования стохастического дифференциального уравнения в банаховом пространстве.

Пусть $X$ - вещественное сепарабельное банахово пространство, $X^{*}$ — сопряженное пространство, $\mathscr{B}(X)$ - борелевская $\sigma$-алгебра в $X$ и $(\Omega, \mathscr{B}, \mathbf{P})$ - вероятностное пространство. Непрерывный линейный оператор $L: X^{*} \rightarrow L_{2}(\Omega, \mathscr{B}, \mathbf{P})$ называется обобщенным случайным элементом (ОСЭ). Рассмотрим такие ОСЭ, которые отображают $X^{*}$ в фиксированное сепарабельное замкнутое подпространство $G \subset L_{2}(\Omega, \mathscr{B}, \mathbf{P})$. Обозначим $\mathscr{M}_{1}:=L\left(X^{*}, G\right)$ - банахово пространство ОСЭ с нормой $\|L\|=\sup _{\left\|x^{*}\right\| \leqslant 1}\left\|L x^{*}\right\|_{L_{2}}$. Мы будем говорить, что случайный элемент (измеримое отображение) $\xi: \Omega \rightarrow X$ имеет слабый второй порядок, если $\mathbf{E}\left\langle\xi, x^{*}\right\rangle^{2}<\infty$ для любого $x^{*} \in X^{*}$. Случайный элемент $\xi$ можно реализовать как элемент пространства $\mathscr{M}_{1}: L_{\xi} x^{*}=\left\langle\xi, x^{*}\right\rangle$ (непрерывность $L_{\xi}$ следует из теоремы о замкнутом графике). Обозначим через $\mathscr{M}_{2}$ линейное нормированное пространство случайных элементов слабого второго порядка с нормой $\|\xi\|=\left\|L_{\xi}\right\|$. Таким образом, мы имеем $\mathscr{M}_{2} \subseteq \mathscr{M}_{1}$. Корреляционным оператором ОСЭ $L \in \mathscr{M}_{1}$ называется отображение $R_{L}: X^{*} \rightarrow X^{* *}, R_{L}=L^{*} L . R_{L}$ - положительно определенный, симметричный, линейный оператор. Известно, что если $L=L_{\xi} \in \mathscr{M}_{2}$, то $R_{L}$ отображает $X^{*}$ в $X$ (см. [2, теорема 3.2.1]), и если $R$ - положительно определенный и симметричный линейный оператор из $X^{*}$ в $X$, то существуют $\left(x_{k}^{*}\right)_{k \in \mathbf{N}} \subset X^{*}$ и $\left(x_{k}\right)_{k \in \mathbf{N}} \subset X$ такие, что $\left\langle R x_{k}^{*}, x_{j}^{*}\right\rangle=\delta_{k j}, R x_{k}^{*}=x_{k}$, $R x^{*}=\sum_{k=1}^{\infty}\left\langle x_{k}, x^{*}\right\rangle x_{k}, x^{*} \in X^{*}$ (см. [2, лемма 3.1.1]). Более того, для $R_{L}: X^{*} \rightarrow X^{* *}$, так как $G$ - сепарабельное подпространство пространства $L_{2}(\Omega, \mathscr{B}, \mathbf{P})$, существуют $\left(x_{k}^{*}\right)_{k \in \mathbf{N}} \subset X^{*}$ и $\left(x_{k}^{* *}\right)_{k \in \mathbf{N}} \subset X^{* *}$ такие, что $\left\langle R x_{k}^{*}, x_{j}^{*}\right\rangle=\delta_{k j}, R x_{k}^{*}=x_{k}^{* *}$ и $R x^{*}=\sum_{k=1}^{\infty}\left\langle x_{k}^{* *}, x^{*}\right\rangle x_{k}^{* *}$ для $x^{*} \in X^{*}$.

Пусть $L \in \mathscr{M}_{1}$. Рассмотрим отображение $m_{L}: X^{*} \rightarrow \mathbf{R}^{1}, m_{L} x^{*}=$ $\mathbf{E} L x^{*}$. Это отображение $m_{L}$ линейно и ограничено (следовательно, $\left.m_{L} \in X^{* *}\right)$ и называется математическим ожиданием ОСЭ $L$. Пусть $L^{\prime} x^{*}=L x^{*}-\left\langle m, x^{*}\right\rangle$. Ковариационным оператором ОСЭ $L$ называется оператор $L^{\prime *} L^{\prime}$. Если $L \in \mathscr{M}_{2}$, т.е. существует $\xi: \Omega \rightarrow X$ такой, что $L x^{*}=\left\langle\xi, x^{*}\right\rangle$, то $m$ принадлежит $X$ (см. [2, теорема 2.3.1]) и является интегралом Петтиса от $\xi$.

Предложение 1. Пусть ОСЭ L принадлежит пространству $\overline{\mathscr{M}}_{2} \subset \mathscr{M}_{1}$. Тогда математическое ожидание $m_{L}$ принадлежит $X u$ 
ковариачионный оператор $R_{L}$ отображает $X^{*}$ в $X$.

Д о к а з а т е л ь с т в о. Пусть $L \in \overline{\mathscr{M}}_{2}$. Тогда существует последовательность $\xi_{n}, n=1,2, \ldots$, такая, что $\left\|L_{\xi_{n}}-L\right\| \rightarrow 0$. Поскольку

$$
\begin{aligned}
\left\|\mathbf{E}\left(\xi_{n}-\xi_{m}\right)\right\| & \leqslant \sup _{\left\|x^{*}\right\| \leqslant 1}\left|\mathbf{E}\left\langle\xi_{n}-\xi_{m}, x^{*}\right\rangle\right| \leqslant \sup _{\left\|x^{*}\right\| \leqslant 1}\left(\mathbf{E}\left\langle\xi_{n}-\xi_{m}, x^{*}\right\rangle^{2}\right)^{1 / 2} \\
& =\left\|\left(\xi_{n}-\xi_{m}\right)\right\|_{\mathscr{M}_{1}} \rightarrow 0,
\end{aligned}
$$

то существует элемент $m \in X$ такой, что для любого $x^{*} \in X^{*}$ выполнено равенство $\mathbf{E} L x^{*}=\left\langle m, x^{*}\right\rangle$. Далее, для удобства изложения, предположим, что $m=0$.

Пусть $R_{n}: X^{*} \rightarrow X$ обозначает ковариационный оператор случайного элемента $\xi_{n}$. По лемме о факторизации (см. [2, лемма 3.1.1]) $R_{n}=L_{\xi_{n}}^{*} L_{\xi_{n}}$, где $L_{\xi_{n}}: X^{*} \rightarrow G_{n}, L_{\xi_{n}}^{*}: G_{n} \rightarrow X$ и $G_{n}$ - замкнутое сепарабельное подпространство пространства $G$. Пусть

$$
\mathscr{H}_{2}:=\overline{L\left(G_{1} \cup G_{2}\right)}, \quad \mathscr{H}_{3}:=\overline{L\left(G_{3} \cup \mathscr{H}_{2}\right)}, \quad \ldots, \quad \mathscr{H}_{n}:=\overline{L\left(G_{n} \cup \mathscr{H}_{n-1}\right)} ;
$$

$\mathscr{H}_{2} \subset \mathscr{H}_{3} \subset \mathscr{H}_{4} \subset \cdots, \mathscr{H}:=\bar{\bigcup}_{n} \mathscr{H}_{n}$. Для любого $n$ можно представить $\mathscr{H}$ как $\mathscr{H}=G_{n} \oplus G_{n}^{\perp}$. Пусть $x^{*} \in X^{*},\left\|L x^{*}-L_{n} x^{*}\right\| \rightarrow 0$ (обозначим $\left.L_{n}:=L_{\xi_{n}}\right)$. Тогда $L x^{*} \in \mathscr{H}$ и

$$
\begin{aligned}
& \left\|R_{n} x^{*}-R_{L} x^{*}\right\|_{X^{* *}}=\left\|L_{n}^{*} L_{n} x^{*}-L^{*} L x^{*}\right\|_{X^{* *}} \\
& \quad \leqslant \sup _{\left\|y^{*}\right\| \leqslant 1}\left|\left\langle L_{n}^{*} L_{n} x^{*}, y^{*}\right\rangle-\left\langle L^{*} L_{n} x^{*}, y^{*}\right\rangle\right|+\sup _{\left\|y^{*}\right\| \leqslant 1}\left|\left\langle L^{*} L_{n} x^{*}, y^{*}\right\rangle-\left\langle L^{*} L x^{*}, y^{*}\right\rangle\right| \\
& \quad \leqslant\left\|L_{n} x^{*}\right\| \sup _{\left\|y^{*}\right\| \leqslant 1}\left\|L_{n} y^{*}-L y^{*}\right\|+\sup _{\left\|y^{*}\right\| \leqslant 1}\left\|L y^{*}\right\|\left\|L_{n} x^{*}-L x^{*}\right\| \rightarrow 0
\end{aligned}
$$

при $n \rightarrow \infty$. Следовательно, $R_{L} x^{*}=\lim _{n \rightarrow \infty} R_{n} x^{*} \in X$, т.е. $R X^{*} \subseteq X$. Предложение доказано.

Предложение 2. Пусть дань симметричнье и положительно определеннье линейнье операторы $R_{1}: X^{*} \rightarrow X^{* *}, R_{2}: X^{*} \rightarrow X$. Если для любого $x^{*} \in X^{*}$ выполнено неравенство $\left\langle R_{1} x^{*}, x^{*}\right\rangle \leqslant\left\langle R_{2} x^{*}, x^{*}\right\rangle$, то $R_{1}$ отображает также $X^{*}$ в $X$.

Д о к а з а т е л ь с т в о. Мы применим технику, использованную при доказательстве леммы о факторизации 3.1 .1 из [2]. Пусть $E_{2}:=$ $\left\{x^{*} \in X^{*}:\left\langle R_{2} x^{*}, x^{*}\right\rangle=0\right\}$. В факторпространстве $X^{*} / E_{2}$ введем скалярное произведение $\left\langle x_{1}^{*}+E_{2}, x_{2}^{*}+E_{2}\right\rangle=\left\langle R_{2} x_{1}^{*}, x_{2}^{*}\right\rangle$. Пусть $H_{2}-$ соответствующее гильбертово пространство и $R_{2}=A_{2}^{*} A_{2}$ - факторизация оператора $R_{2}, A_{2}: X^{*} \rightarrow H_{2}$. Аналогично, для оператора $R_{1}$ введем $E_{1}$ и $H_{1}, R_{1}=A_{1}^{*} A_{1}, A_{1}: X^{*} \rightarrow H_{1}$. Так как $\left\langle R_{1} x^{*}, x^{*}\right\rangle \leqslant\left\langle R_{2} x^{*}, x^{*}\right\rangle$, $\operatorname{ker} R_{1} \subseteq \operatorname{ker} R_{2}$, отображение $I_{0}: X^{*} / E_{2} \rightarrow X^{*} / E_{1}$ ограничено, ограничено и $I: H_{2} \rightarrow H_{1}$. Таким образом, $R_{1}=A_{1}^{*} A_{1}=A_{2}^{*} I^{*} I A_{2}$. Следовательно, $R_{1}\left(X^{*}\right) \subset X$. Предложение доказано. 
Предложение 3. Пусть дан $О С \ni L \in \mathscr{M}_{1}$. Предположим, что $\mathbf{E} L=0$ и ковариачионньіи оператор $R_{L}: X^{*} \rightarrow X^{* *}$ равен $R_{L} x^{*}=$ $\sum_{k=1}^{\infty}\left\langle a_{k}^{* *}, x^{*}\right\rangle a_{k}^{* *}$, где $\left(a_{k}^{*}\right)_{k \in \mathbf{N}},\left(a_{k}^{* *}\right)_{k \in \mathbf{N}}$ maкue, uто $R_{L} a_{k}^{*}=a_{k}^{* *},\left\langle a_{k}^{* *}, a_{j}^{*}\right\rangle=$ $\delta_{k j}$. Тогда $L x^{*}=\sum_{k=1}^{\infty}\left\langle a_{k}^{* *}, x^{*}\right\rangle L a_{k}^{*}$.

Д ок аз а т е ль с т в о. Для любого $x^{*} \in X^{*}$ выполнены равенства $\mathbf{E}\left(L x^{*}\right)^{2}=\left\langle R_{L} x^{*}, x^{*}\right\rangle=\sum_{k=1}^{\infty}\left\langle a_{k}^{* *}, x^{*}\right\rangle^{2}$. Имеем

$$
\begin{aligned}
& \mathbf{E}\left(L x^{*}-\sum_{k=1}^{n}\left\langle a_{k}^{* *}, x^{*}\right\rangle L a_{k}^{*}\right)^{2} \\
& =\mathbf{E}\left(L x^{*}\right)^{2}-2 \sum_{k=1}^{n}\left\langle a_{k}^{* *}, x^{*}\right\rangle \mathbf{E} L x^{*} L a_{k}^{*}+\mathbf{E}\left(\sum_{k=1}^{n}\left\langle a_{k}^{* *}, x^{*}\right\rangle L a_{k}^{*}\right)^{2} \\
& =\left\langle R_{L} x^{*}, x^{*}\right\rangle-2 \sum_{k=1}^{n}\left\langle a_{k}^{* *}, x^{*}\right\rangle\left\langle R_{L} a_{k}^{*}, x^{*}\right\rangle+\sum_{k=1}^{n}\left\langle a_{k}^{* *}, x^{*}\right\rangle^{2} \\
& =\sum_{k=n+1}^{\infty}\left\langle a_{k}^{* *}, x^{*}\right\rangle^{2} \rightarrow 0 .
\end{aligned}
$$

Предложение доказано.

2. Стохастический интеграл. Пусть $\left(W_{t}\right)_{t \in[0,1]}$ - одномерный винеровский процесс и $\left(\mathscr{F}_{t}\right)_{t \in[0,1]}$ - неубывающее семейство $\sigma$-алгебр такое, что: а) $W_{t}$ является $\mathscr{F}_{t}$-измеримым для любого $t \in[0,1]$; b) $W_{s}-W_{t}$ не зависит от $\sigma$-алгебры $\mathscr{F}_{t}$ для всех $s>t ; \mathscr{F}_{t}$ пополнена $\mathbf{P}$-нуль множествами из $\mathscr{B}$. Пусть $\left(L_{t}\right)_{t \in[0,1]}$ является семейством ОСЭ; мы назовем его обобщенным случайным процессом (ОСП). Если $\left(\xi_{t}\right)_{t \in[0,1]}$, $\xi_{t}: \Omega \rightarrow X,-$ случайный процесс слабого второго порядка, то он порождает ОСП $L_{\xi_{t}} x^{*}=\left\langle\xi_{t}, x^{*}\right\rangle$.

О п р е д е л е н и е 1 . ОСП $\left(L_{t}\right)_{t \in[0,1]}$ называется неупреждающим относительно семейства $\sigma$-алгебр $\left(\mathscr{F}_{t}\right)_{t \in[0,1]}$, если для любого $x^{*} \in X^{*}$ числовой случайный процесс $L_{t} x^{*}$ является $([0,1] \times \Omega, \mathscr{B}[0,1] \times \mathscr{B})$ измеримым и для любого $t \in[0,1], L_{t} x^{*} \mathscr{F}_{t}$-измерим. Обозначим через $T \mathscr{M}_{1}$ банахово пространство неупреждающих ОСП $\left(L_{t}\right)_{t \in[0,1]}$, для котоpыx

$$
\left\|L_{t}\right\|:=\sup _{\left\|x^{*}\right\| \leqslant 1}\left(\int_{0}^{1} \mathbf{E}\left(L_{t} x^{*}\right)^{2} d t\right)^{1 / 2}<\infty .
$$

Если $\xi_{t}: \Omega \rightarrow X, t \in[0,1],-$ случайный процесс такой, что для всех $x^{*} \in X^{*}$ процесс $\left\langle\xi_{t}, x^{*}\right\rangle_{t \in[0,1]}$ неупреждающий и $\int_{0}^{1} \mathbf{E}\left\langle\xi_{t}, x^{*}\right\rangle^{2} d t<\infty$, то $L_{\xi_{t}} \in T \mathscr{M}_{1}$.

Для любого $x^{*} \in X^{*}$ мы можем определить стохастический интеграл $\int_{0}^{1} L_{t} x^{*} d W_{t}$. 
О п р е д е л е н и е 2. Для любого неупреждающего ОСП $\left(L_{t}\right)_{t \in[0,1]} \in$ T㶡 оператор

$$
I(L): X^{*} \rightarrow L_{2}(\Omega, \mathscr{B}, \mathbf{P}), \quad I(L) x^{*}=\int_{0}^{1} L_{t} x^{*} d W_{t},
$$

называется обобщенным стохастическим интегралом (ОСИ) от $\left(L_{t}\right)_{t \in[0,1]}$.

Нетрудно видеть, что $I(L) \in \mathscr{M}_{1}$.

Предложение 4. Оператор стохастического интегрирования $I: T \mathscr{M}_{1} \rightarrow \mathscr{M}_{1}$ является изометрическим оператором; для любого $\left(L_{t}\right)_{t \in[0,1]} \in T \mathscr{M}_{1}$ справедливь равенства $\mathbf{E} I(L)=0 \in X^{* *} u$ $\left\langle R_{I(L)} x^{*}, y^{*}\right\rangle=\mathbf{E} I(L) x^{*} I(L) y^{*}=\left\langle R_{L} x^{*}, y^{*}\right\rangle$, где $R_{L}$ sвляется отображением из $X^{*}$ в $X^{* *} u\left\langle R_{L} x^{*}, y^{*}\right\rangle=\int_{0}^{1} \int_{\Omega} L_{t} x^{*} L_{t} y^{*} d \mathbf{P} d t$.

Д о к а з а т е л ь с т в о. Поскольку $\mathbf{E} \int_{0}^{1} L_{t} x^{*} d W_{t}$ является линейным ограниченным функционалом из $X^{*}$ в $\mathbf{R}^{1}$, то он принадлежит $X^{* *}$ и равен нулю. Имеем

$$
\|I(L)\|_{\mathscr{M}_{1}}^{2}=\sup _{\left\|x^{*}\right\| \leqslant 1} \mathbf{E}\left(\int_{0}^{1} L_{t} x^{*} d W_{t}\right)^{2}=\sup _{\left\|x^{*}\right\| \leqslant 1} \mathbf{E} \int_{0}^{1}\left(L_{t} x^{*}\right)^{2} d t=\|L\|_{T \mathscr{M}_{1}}^{2} .
$$

Все остальные равенства следуют из определения корреляционного оператора ОСЭ.

О п р е д е л е н и е 3. Стохастическим интегралом от ОСП $\left(L_{t}\right)_{t \in[0,1]}$ называется $X$-значный случайный элемент $\eta: \Omega \rightarrow X$ (если такой элемент существует), для которого $\left\langle\eta, x^{*}\right\rangle=I(L) x^{*}$ п.н. для любого $x^{*} \in X^{*}, \eta:=\int_{0}^{1} L_{t} d W_{t}$.

Очевидно, что если $T$ неслучайна, то $m^{* *}:=T \in X^{* *}$. Приведем простой пример, когда $m^{* *}(t) \notin X$, но стохастический интеграл $\xi:=$ $\int_{0}^{1} m^{* *}(t) d W_{t}, \xi: \Omega \rightarrow X$, существует. Пусть $X=c_{0}$ и

$$
e_{k, n}(t)=\left\{\begin{array}{ll}
1, & t \in\left[\frac{k-1}{n}, \frac{k}{n}\right], \\
0, & t \notin\left[\frac{k-1}{n}, \frac{k}{n}\right],
\end{array} \quad n=1,2, \ldots, \quad k=1, \ldots, n .\right.
$$

Ясно, что $e(t):=\left(e_{k, n}(t)\right)_{k \leqslant n, n \in \mathbf{N}}, t \in[0,1]$, не принадлежит пространству $c_{0}$, но $e(t) \in l_{\infty}, t \in[0,1]$. Рассмотрим отображение

$$
L(t): l_{1} \rightarrow \mathbf{R}^{1}, \quad L(t)(\vec{\lambda})=\sum_{n=1}^{\infty} \sum_{k=1}^{n} \lambda_{n, k} e_{n, k}(t),
$$

где $\vec{\lambda}:=\left(\lambda_{n, k}\right)_{n \in \mathbf{N}, k \leqslant n} \in l_{1}$. Имеем

$$
\int_{0}^{1}\left\langle L_{t}, \vec{\lambda}\right\rangle d W_{t}=\sum_{n=1}^{\infty} \sum_{k=1}^{n} \lambda_{n, k}\left(W_{k / n}-W_{(k-1) / n}\right)=\langle\vec{\lambda}, \xi\rangle,
$$


где $\xi:=\left(W_{k / n}-W_{(k-1) / n}\right)_{k \leqslant n, n \in \mathbf{N}}$. Пусть $\sigma_{n, k}:=\mathbf{E}\left(W_{k / n}-W_{(k-1) / n}\right)^{2}$. Так как

$$
\sum_{n=1}^{\infty} \sum_{k=1}^{n} \exp \left(-\frac{\mu}{\sigma_{k, n}}\right)=\sum_{n=1}^{\infty} n \exp (-\mu n)<\infty
$$

для любого положительного $\mu$, то $\xi$ является случайным элементом в банаховом пространстве $c_{0}$ (см. [2, предложение 5.5.8]). Следовательно, стохастический интеграл $\int_{0}^{1} L_{t} d W_{t}$ существует.

Для любых $L:=\left(L_{t}\right)_{t \in[0,1]} \in T \mathscr{M}_{1}$ и $t \in[0,1]$ имеем $L I_{[0, t]} \in T \mathscr{M}_{1}$. Следовательно, можно определить $I(L)_{t} \in T \mathscr{M}_{1}: I(L)_{t} x^{*}:=\int_{0}^{t} L_{s} x^{*} d W_{s}$. Для любого $L \in T \mathscr{M}_{1}$ ОСЭ $I(L)_{t}$ существует, но из существования стохастического интеграла $\xi: \Omega \rightarrow X,\left\langle\xi, x^{*}\right\rangle=\int_{0}^{1} L_{t} x^{*} d W_{t}$, не следует существование стохастического интеграла $\int_{0}^{t} L_{s} d W_{s}=\xi_{t}=\mathbf{E}\left(\xi \mid \mathscr{F}_{t}\right)$.

\section{3. Достаточные условия существования стохастического} интеграла. Пусть

$$
\begin{gathered}
\left(L_{t}\right)_{t \in[0,1]} \in T \mathscr{M}_{1}, \\
m^{* *}(t):=\mathbf{E} L_{t}, \\
L_{t}^{1}:=L_{t}-m^{* *}(t) .
\end{gathered}
$$

Мы будем отдельно рассматривать стохастические интегралы от $m^{* *}(t)$ (от неслучайной функции) и от ОСП $L^{1}$ с нулевым математическим ожиданием.

Теорема 1. Пусть неслучайная функция $m^{* *}(t)$ отображает $[0,1]$ в $X^{* *}$ u $\int_{0}^{1}\left\langle m^{* *}(t), x^{*}\right\rangle^{2} d t<\infty$ для любого $x^{*} \in X^{*}$. Тогда стохастический интеграл $\int_{0}^{1} m^{* *}(t) d W_{t}$ существует в том и только том случае, если $R x^{*}=\int_{0}^{1}\left\langle m^{* *}(t), x^{*}\right\rangle m^{* *}(t) d t$ отображает $X^{*}$ в $X$ u $R$ есть гауссовская ковариачия. Если $R x^{*}=\sum_{k=1}^{\infty}\left\langle a_{k}, x^{*}\right\rangle a_{k},\left(a_{k}\right)_{k \in \mathbf{N}} \subset X$ $u\left(a_{k}^{*}\right)_{k \in \mathbf{N}} \subset X^{*}$ таковьи, ито $R a_{k}^{*}=a_{k} u\left\langle R a_{k}^{*}, a_{j}^{*}\right\rangle=\delta_{k j}$, то ряд $\sum_{k=1}^{\infty} a_{k} \int_{0}^{t}\left\langle m^{* *}(s), a_{k}\right\rangle d W_{s}$ сходится равномерно по $t$ n.н. $\kappa$ стохастическому интегралу $I_{t}=\int_{0}^{t} m^{* *}(s) d W_{s}$ u, следовательно, $\left(I_{t}\right)_{t \in[0,1]}$ имеет п.н. непрерьвнье реализачии.

Д о к а з а т е ль с т в о. Необходимость очевидна, так как если стохастический интеграл существует, то он является гауссовским случайным элементом. Следовательно, $R$ есть гауссовская ковариация. Достаточность также легко доказывается: пусть

$$
\begin{gathered}
L: X^{*} \rightarrow L_{2}(\Omega, \mathscr{B}, \mathbf{P}), \\
L x^{*}=\int_{0}^{1}\left\langle m^{* *}(t), x^{*}\right\rangle d W_{t} .
\end{gathered}
$$

Тогда $L x^{*}$ - гауссовский ОСЭ, $R_{L}: X^{*} \rightarrow X-$ гауссовская ковариация. Следовательно, существует гауссовский случайный элемент $\xi: \Omega \rightarrow X$ такой, что $\int_{0}^{1}\left\langle m^{* *}(t), x^{*}\right\rangle d W_{t}=\left\langle\xi, x^{*}\right\rangle$, т.е. существует стохастический 
интеграл $\int_{0}^{1} m^{* *}(t) d W_{t}$. Далее, $\int_{0}^{1}\left\langle m^{* *}(t), a_{k}^{*}\right\rangle d W_{t}, k=1,2, \ldots$, - гауссовские стандартные случайные величины. По теореме Ито-Нисио, ряд $\sum_{k=1}^{\infty} a_{k} \int_{0}^{1}\left\langle m^{* *}(t), a_{k}^{*}\right\rangle d W_{t}$ сходится в $X$ к стохастическому интегралу $\int_{0}^{1} m^{* *}(t) d W_{t}$, так как для любого $x^{*} \in X^{*}$ имеем

$$
\begin{gathered}
\mathbf{E}\left(\int_{0}^{1}\left\langle m^{* *}(t), x^{*}\right\rangle d W_{t}-\sum_{k=1}^{n}\left\langle a_{k}, x^{*}\right\rangle \int_{0}^{1}\left\langle m^{* *}(t), a_{k}^{*}\right\rangle d W_{t}\right)^{2} \\
=\sum_{k=n+1}^{\infty}\left\langle a_{k}, x^{*}\right\rangle^{2} \rightarrow 0, \quad n \rightarrow \infty .
\end{gathered}
$$

Докажем, что ряд $\sum_{k=1}^{\infty} a_{k} \int_{0}^{t}\left\langle m^{* *}(s), a_{k}\right\rangle d W_{s}$ сходится равномерно по $t$ в $X$. Пусть $\zeta_{n}(t, \omega):=\sum_{k=1}^{n} a_{k} \int_{0}^{t}\left\langle m^{* *}(s), a_{k}\right\rangle d W_{s}$, тогда $\zeta_{n}(\cdot, \omega) \in$ $C([0,1], X)$. Так как $\sum_{k=n}^{m} a_{k} \int_{0}^{t}\left\langle m^{* *}(s), a_{k}^{*}\right\rangle d W_{s}$ является процессом с независимыми приращениями и с непрерывными реализациями, то по неравенству Леви

$$
\begin{aligned}
\mathbf{P}\left(\sup _{t \in[0,1]}\left\|\sum_{k=n}^{m} a_{k} \int_{0}^{t}\left\langle m^{* *}(s), a_{k}^{*}\right\rangle d W_{s}\right\|>\varepsilon\right) \\
\quad \leqslant 2 \mathbf{P}\left(\left\|\sum_{k=n}^{m} a_{k} \int_{0}^{1}\left\langle m^{* *}(s), a_{k}^{*}\right\rangle d W_{s}\right\|>\varepsilon\right) \rightarrow 0,
\end{aligned}
$$

т.е. $\sum a_{k} \int_{0}^{t}\left\langle m^{* *}(s), a_{k}^{*}\right\rangle d W_{s}$ сходится по мере в $C([0,1], X)$. По теореме Ито-Нисио, имеем п.н. сходимость в $C([0,1], X)$. Следовательно, сумма является процессом с п.н. непрерывными реализациями. Ввиду того, что

$$
\sum_{k=1}^{\infty} a_{k} \int_{0}^{1}\left\langle m^{* *}(t), a_{k}\right\rangle d W_{t}=\int_{0}^{1} m^{* *}(t) d W_{t}
$$

и

$\sum_{k=1}^{\infty} a_{k} \int_{0}^{t}\left\langle m^{* *}(s), a_{k}^{*}\right\rangle d W_{s}=\mathbf{E}\left(\int_{0}^{1} m^{* *}(s) d W_{s} \mid \mathscr{F}_{t}\right)=\int_{0}^{t} m^{* *}(s) d W_{s}=I_{t}$,

получаем, что процесс $\left(I_{t}\right)_{t \in[0,1]}$ имеет п.н. непрерывные реализации. Теорема доказана.

3 а м е ч а н и е. Необходимое и достаточное условие существования стохастического интеграла от неслучайной функции было получено в [7]. Там был определен стохастический интеграл как предел последовательности стохастических интегралов от ступенчатых функций.

Следующая теорема дает достаточное условие принадлежности ОСИ пространству $\overline{\mathscr{M}}_{2}$ и представляет такие ОСИ как предел последовательности стохастических интегралов от неупреждающих ступенчатых функций. 
Теорема 2. Пусть неупреждающий ОСП $L:[0,1] \rightarrow \mathscr{M}_{1}$ таков, что $L(t) \in \overline{\mathscr{M}_{2}}$, множество $(L(t), t \in[0,1])$ сепарабельно в $\overline{\mathscr{M}_{2}} u$ $\int_{0}^{1}\|L(t)\|_{\mathscr{M}_{1}}^{2} d t<\infty$. Тогда существует последовательность неупреждающих ступенчатых функиии $f_{n}:[0,1] \times \Omega \rightarrow X$ такая, что $\int_{0}^{1}\left\|f_{n}(t)-L(t)\right\|_{\mathscr{M}_{1}}^{2} d t \rightarrow 0 u\left\|\int_{0}^{1} f_{n}(t) d W(t)-\int_{0}^{1} L(t) d W(t)\right\|_{\mathscr{M}_{1}}^{2} \rightarrow 0, u$, следовательно, $\int_{0}^{1} L(t) d W(t) \in \overline{\mathscr{M}}_{2}$.

Д ок а з а т е ль с т в о. Нетрудно заметить, что без ограничения общности можно считать, что $\|L(t)\|_{\mathscr{M}_{1}} \leqslant C$. Если $L:[0,1] \rightarrow \mathscr{M}_{1}$ непрерывен, то $\left\|L(t)-\sum_{k=0}^{n-1} L(k / n) I_{(k / n,(k+1) / n]}\right\|_{\mathscr{M}_{1}} \rightarrow 0$ при $n \rightarrow \infty$. Так как $\left\|\sum_{k=0}^{n-1} L(k / n) I_{(k / n,(k+1 / n)]}\right\|_{\mathscr{M}_{1}} \leqslant C$, то по теореме Лебега $\int_{0}^{1}\left\|L(t)-L_{n}(t)\right\|_{\mathscr{M}_{1}} d t \rightarrow 0$, где $L_{n}(t):=\sum_{k=0}^{n-1} L(k / n) I_{(k / n,(k+1) / n]}$. Так как $L(t) \in \overline{\mathscr{M}}_{2}$, то существует последовательность неупреждающих ступенчатых функций $f_{n}:[0,1] \times \Omega \rightarrow X$ такая, что $\int_{0}^{1}\left\|L_{n}-f_{n}\right\|_{\mathscr{M}_{1}}^{2} d t \leqslant 1 / n$. Это есть искомая последовательность. Пусть теперь $L-$ произвольный ОСП. Для любого $x^{*} \in X^{*}$ и $g \in G, L(t) x^{*} g$ измерима, и так как $f\left(x^{*}, g\right): \mathscr{M}_{1} \rightarrow \mathbf{R}^{1}, f(T)=\int_{\Omega} T x^{*} g d \mathbf{P}$, отделяет точки в $\mathscr{M}_{1}$ и процесс $L:[0,1] \rightarrow \mathscr{M}_{1}$ сепарабельнозначный, то по теореме Петтиса (см. [2, предложение 1.1.10]) $L:[0,1] \rightarrow \mathscr{M}_{1}$ измерим. Так как $L$ ограничена, то существует интеграл Бохнера $\int_{s}^{t} L(u) d u$, который принадлежит $\overline{\mathscr{M}}_{2}$. Пусть $L_{m}(t):=m \int_{(t-1 / m) \vee 0}^{t} L(s) d s$. Тогда $L_{m}(t) \rightarrow L(t)$ п.в. по $t$ при $n \rightarrow \infty$ (см. [3, следствие 2 , теорема 3.8.5]). По теореме Лебега $\int_{0}^{1}\left\|L_{m}(t)-L(t)\right\|_{\mathscr{M}_{1}}^{2} d t \rightarrow 0$. Так как $L_{m}(t)$ непрерывно для любого $m$, то существует последовательность неупреждающих ступенчатых функций $f_{m, n}(t):[0,1] \times \Omega \rightarrow X$ такая, что $\int_{0}^{1}\left\|f_{m, n}-L_{m}\right\|_{\mathscr{M}_{1}}^{2} d t \rightarrow 0$ при $n \rightarrow \infty$. Тогда $\int_{0}^{1}\left\|f_{m, n}(t)-L(t)\right\|_{\mathscr{M}_{1}}^{2} d t \rightarrow 0$ и

$$
\begin{gathered}
\left\|\int_{0}^{1} L(t) d W(t)-\int_{0}^{1} f_{m, n} d W(t)\right\|_{\mathscr{M}_{1}}^{2}=\sup _{\left\|x^{*}\right\| \leqslant 1} \mathbf{E} \int_{0}^{1}\left(L(t)-\left\langle f_{m, n}(t), x^{*}\right\rangle\right)^{2} d t \\
\quad \leqslant \int_{0}^{1} \sup _{\left\|x^{*}\right\| \leqslant 1} \mathbf{E}\left(L(t)-\left\langle f_{m, n}(t), x^{*}\right\rangle\right)^{2} d t=\int_{0}^{1}\left\|L(t)-f_{m, n}(t)\right\|_{\mathscr{M}_{1}}^{2} d t \rightarrow 0 .
\end{gathered}
$$

Следовательно, $\int_{0}^{1} L(t) d W(t) \in \overline{\mathscr{M}_{2}}$. Теорема доказана.

Отсюда мы имеем следующее утверждение.

Следствие 1. Пусть случайный прочесс $f:[0,1] \times \Omega \rightarrow X$ таков, uто $f(\cdot, w)$, как отображение из $[0,1]$ в пространство $M_{1}$, сепарабельнозначное и $\int_{0}^{1}\|f(t)\|_{\mathscr{M}_{1}}^{2} d t<\infty$. Тогда существует последовательность неупреждающих ступенчатьхх функиий $\left(f_{n}\right)_{n \in \mathbf{N}}, f_{n}:[0,1] \rightarrow X$, такая, что $\int_{0}^{1}\left\|f(t)-f_{n}(t)\right\|_{\mathscr{M}_{1}}^{2} d t \rightarrow 0, u$, следовательно, $\int_{0}^{1} f(t) d W(t) \in$ $\overline{\mathscr{M}_{2}}$.

Для получения достаточных условий существования стохастических интегралов рассмотрим положительно определенные симметричные операторы $R: X^{*} \rightarrow X$. 
О п р е д е л е н и е 4. Будем говорить, что положительно определенный симметричный оператор $R: X^{*} \rightarrow X$ принадлежит $\mathbf{R}_{p}(X)$, если в факторизации $R=A A^{*}$ оператор $A: H \rightarrow X$ является $p$-абсолютно суммирующим, т.е. существует $c>0$ такое, что для любых $n \in \mathbf{N}$ и $h_{1}, \ldots, h_{n}$ из $H$

$$
\left(\sum_{i=1}^{n}\left\|A h_{i}\right\|^{p}\right)^{1 / p} \leqslant c \sup _{\|h\| \leqslant 1}\left(\sum_{i=1}^{n}\left\langle h_{i}, h\right\rangle^{p}\right)^{1 / p} .
$$

Обозначим через $\Pi_{p}(R)$ минимальное значение $c$, для которого выполнено неравенство (1).

Из леммы о факторизации легко вывести следующее предложение.

Предложение 5. Положительно определенный симметричный опеpатор $R: X^{*} \rightarrow X$ принадлежит $\mathbf{R}_{p}(X)$ тогда и только тогда, когда существует $c>0$ такое, что для любых $n \in \mathbf{N} u x_{1}^{*}, \ldots, x_{n}^{*}$ из $X^{*}$

$$
\left(\sum_{i=1}^{n}\left\|R x_{i}^{*}\right\|^{p}\right)^{1 / p} \leqslant c \sup _{\left\langle R x^{*}, x^{*}\right\rangle \leqslant 1}\left(\sum_{i=1}^{n}\left|\left\langle R x_{i}^{*}, x^{*}\right\rangle\right|^{p}\right)^{1 / p} .
$$

Минимальное с, для которого справедливо неравенство (2), равно $\Pi_{p}(R)$.

Нам понадобится следующая лемма.

Лемма 1. Пусть дань положительно определенные симметричнье операторы $R_{1}$ и $R_{2}$ такие, что для любого $x^{*} \in X^{*}$ выполнено неравенство $\left\langle R_{1} x^{*}, x^{*}\right\rangle \leqslant\left\langle R_{2} x^{*}, x^{*}\right\rangle$. Eсли $R_{2} \in \mathbf{R}_{p}(X)$, то $R_{1} \in \mathbf{R}_{p}(X)$.

Д ок а з а т е л ь с т в о. При доказательстве предложения 2 было показано, что если $R_{2}=A A^{*}$, то $R_{1}=A J J^{*} A^{*}$. Пусть $A$ есть $p$ абсолютно суммирующий оператор, тогда $A J$ тоже будет $p$-абсолютно суммирующим оператором, т.е. $R_{1} \in \mathbf{R}_{p}(X)$. Лемма доказана.

Следующая лемма дает оценку сильного $p$-го момента случайного элемента $\mathbf{R}_{p}$-нормой его ковариационного оператора.

Лемма 2. Пусть дан случайный элемент слабого второго порядка $\xi: \Omega \rightarrow X$ такой, что $R_{\xi} x^{*}=\mathbf{E}\left\langle\xi, x^{*}\right\rangle \xi$ принадлежит $\mathbf{R}_{p}(X)$ u $\left(\mathbf{E}\left|\left\langle\xi, x^{*}\right\rangle\right|^{p}\right)^{1 / p} \leqslant C\left(\mathbf{E}\left\langle\xi, x^{*}\right\rangle^{2}\right)^{1 / 2}$ для некоторого $C>0$ и любого $x^{*} \in X$. Тогда $\mathbf{E}\|\xi\|^{p} \leqslant \Pi_{p}^{p}\left(R_{\xi}\right)$.

Доказательство этой леммы аналогично доказательству леммы 2 из [5] для случая $p=2$.

Приведем достаточные условия существования стохастического интеграла от неупреждающих ОСП. Как и ранее, мы считаем, что математическое ожидание ОСП равно нулю.

Теорема 3. Пусть дан неупреждающий OCП $\left(L_{t}\right)_{t \in[0,1]} \in T \mathscr{M}_{1}$, $\mathbf{E} L_{t}=0$, такой, что оператор $R x^{*}=\int_{0}^{1} \int_{\Omega} L_{t} x^{*} L_{t} d \mathbf{P} d t$ отображает 
$X^{*}$ в $X$ и для некоторого $p \geqslant 2$ выполнены включение $R \in \mathbf{R}_{p}(X)$ u неравенство $\left(\mathbf{E} \int_{0}^{1}\left|L_{t} x^{*}\right|^{p} d t\right)^{1 / p} \leqslant \lambda(p)\left(\mathbf{E} \int_{0}^{1}\left(L_{t} x^{*}\right)^{2} d t\right)^{1 / 2}$. Тогда существует стохастический интеграл

$$
I_{t}=\int_{0}^{t} L_{s} d W_{s}=\sum_{k=1}^{\infty} x_{k} \int_{0}^{t} L_{s} x_{k}^{*} d W_{s}
$$

Ряд в правой части равенства (3) сходится в $L_{p}(\Omega, X), \mathbf{E}\left\|I_{t}\right\|^{p}<\infty$ $u\left(I_{t}\right)_{t \in[0,1]}$ имеет п.н. непрерыьвные реализаиии, где $\left(x_{k}\right)_{k \in \mathbf{N}} u\left(x_{k}^{*}\right)_{k \in \mathbf{N}}$ maкue, ито $R x_{k}^{*}=x_{k},\left\langle R x_{k}^{*}, x_{j}^{*}\right\rangle=\delta_{k j}, k, j=1,2, \ldots, u R x^{*}=$ $\sum_{k=1}^{\infty}\left\langle x_{k}, x^{*}\right\rangle x_{k}$.

Д о к а з а т е ль с т в о. Предположим, что $R=A A^{*}, A: H \rightarrow X$ и $\left(e_{k}\right)_{k \in \mathbf{N}} \subset H$ таковы, что $A e_{k}=x_{k}, k=1,2, \ldots$ Рассмотрим $\zeta_{n}(t)=\sum_{k=1}^{n} e_{k} \int_{0}^{1} L x_{k}^{*} d W_{t}$ : для любого $h \in H,\left(\left\langle\zeta_{n}(t), h\right\rangle\right)_{n \in \mathbf{N}}$ сходится в $L_{p}(\Omega, \mathscr{B}, \mathbf{P})$, так как

$$
\begin{aligned}
& \left(\mathbf{E}\left|\left\langle\sum_{k=n}^{m} e_{k} \int_{0}^{1} L_{t} x_{k}^{*} d W_{t}, h\right\rangle\right|^{p}\right)^{1 / p}=\left(\mathbf{E}\left|\int_{0}^{1} L_{t}\left(\sum_{k=n}^{m} h_{k} x_{k}^{*}\right) d W_{t}\right|^{p}\right)^{1 / p} \\
& \leqslant c_{p}\left(\mathbf{E} \int_{0}^{1}\left|L_{t}\left(\sum_{k=n}^{m} h_{k} x_{k}^{*}\right)\right|^{p} d t\right)^{1 / p} \leqslant c_{p} \lambda_{p}\left(\mathbf{E} \int_{0}^{1}\left(L_{t}\left(\sum_{k=n}^{m} h_{k} x_{k}^{*}\right)^{2} d t\right)^{1 / 2}\right. \\
& =c_{p} \lambda_{p}\left(\sum_{k=n}^{m} h_{k}^{2}\right)^{1 / 2} \rightarrow 0, \quad n, m \rightarrow \infty
\end{aligned}
$$

По лемме 6.5.2 из [2] $A \zeta_{n}=\sum_{k=1}^{n} x_{k} \int_{0}^{1} L_{t} x_{k}^{*} d W_{t}$ сходится в $L_{p}(\Omega, X)$. Следовательно, $\mathbf{E}\left\|I_{t}\right\|^{p}<\infty$.

Для доказательства п.н. непрерывности реализаций мы воспользуемся методом, приведенным в [10, теорема 2.1.6]. Пусть $\left(y_{k}^{*}\right)_{k \in \mathbf{N}}-$ тотальное множество в $X^{*}$. Существует $\Omega_{0} \subset \Omega, \mathbf{P}\left(\Omega_{0}\right)=1$ такое, что $\left\langle I_{t}(\omega), y_{n}\right\rangle$ и $\zeta_{n}(t, \omega), n=, 2, \ldots$, непрерывны для всех $\omega \in \Omega_{0}$. Для $t_{m} \rightarrow t$ и $\omega \in \Omega_{0}$

$$
\liminf _{t_{m} \rightarrow t}\left|\left\langle I_{t_{m}}(\omega)-\zeta_{n}\left(t_{m}, \omega\right), y_{k}^{*}\right\rangle\right| \geqslant\left|\left\langle I_{t}(\omega)-\zeta_{n}(t, \omega), y_{k}^{*}\right\rangle\right| .
$$

Следовательно,

$$
\liminf _{t_{m} \rightarrow t}\left\|I_{t_{m}}(\omega)-\zeta_{n}\left(t_{m}, \omega\right)\right\| \geqslant\left\|I_{t}(\omega)-\zeta_{n}(t, \omega)\right\| .
$$

Тогда

$$
\sup _{t \in[0,1]}\left\|I_{t}(\omega)-\zeta_{n}(t, \omega)\right\|=\sup _{t \in \mathbf{Q}}\left\|I_{t}(\omega)-\zeta_{n}(t, \omega)\right\|, \quad \omega \in \Omega_{0},
$$

где $\mathbf{Q}-$ множество рациональных чисел в $[0,1]$. Так как $\left\|I_{t}-\zeta_{n}(t)\right\|-$ субмартингал, то для любого $\varepsilon>0$

$$
\mathbf{P}\left(\sup _{t \in \mathbf{Q}}\left\|I_{t}-\zeta_{n}(t)\right\|>\varepsilon\right) \leqslant \varepsilon^{-1} \mathbf{E}\left\|I_{1}-\zeta_{n}(1)\right\| \rightarrow 0, \quad n \rightarrow \infty .
$$


Тогда существует последовательность $\left(n_{k}\right)_{k \in \mathbf{N}}$ такая, что

$$
\lim _{n_{k} \rightarrow \infty} \sup _{t \in[0,1]}\left\|I_{t}-\zeta_{n_{k}}(t)\right\|=0 \text { п.н. }
$$

Следовательно, $I_{t}$ имеет п.н. непрерывные реализации. Теорема доказана.

Рассмотрим следующий класс ОСЭ. ОСЭ $L \in \mathscr{M}_{1}, \mathbf{E} L=0$, принадлежит $G_{\Phi} \mathscr{M}_{1}$-классу, если существует функция $\Phi: \mathbf{R}_{+} \rightarrow \mathbf{R}_{+}$такая, что $\left(\mathbf{E}\left|L x^{*}\right|^{p}\right)^{1 / p} \leqslant \Phi(p)\left(\mathbf{E}\left(L x^{*}\right)^{2}\right)^{1 / 2}$. Если $\Phi(p)=\sqrt{p}$, то класс $G_{\Phi} \mathscr{M}_{1}$ содержит гауссовские ОСЭ; более точно, он содержит строго субгауссовские ОСЭ. Обозначим через $G_{\Phi} \mathscr{M}_{2}$ класс соответствующих случайных элементов; $G_{\Phi} \mathscr{M}_{2} \subset G_{\Phi} \mathscr{M}_{1}$.

Следствие 2. Пусть дан неупреждающий $O C П\left(L_{t}\right)_{t \in[0,1]} \in T \mathscr{M}_{1}$ такой, ито для некоторого $\Phi: \mathbf{R}^{+} \rightarrow \mathbf{R}^{+}$

$$
L_{t} \in G_{\Phi} \mathscr{M}_{1}, \quad t \in[0,1] .
$$

Если $R x^{*}=\int_{0}^{1} \int_{\Omega} L_{t}^{*} L_{t} x^{*} d \mathbf{P} d t$ отображает $X^{*}$ в $X, R \in \mathbf{R}_{p}(X) u$ $\left.\int_{0}^{1}\left\langle R_{t} x^{*}, x^{*}\right\rangle^{p / 2} d t\right)^{2 / p} \leqslant C_{p}\left\langle R x^{*}, x^{*}\right\rangle$ для каких-нибудь $p \geqslant 2 u C_{p}>0$, то существует стохастический интеграл $\xi=\int_{0}^{1} L_{t} d W_{t}$.

Теорема 4. Пусть неупреждающий ОСП $\left(\xi_{t}\right)_{t \in[0,1]} \in T \mathscr{M}_{2}$ является мартингалом относительно семейства $\sigma$-алгебр $\left(\mathscr{F}_{t}\right)_{t \in[0,1]}$, ковариачионный оператор $R_{1}: X^{*} \rightarrow X, R_{1} x^{*}=\mathbf{E}\left\langle\xi_{1}, x^{*}\right\rangle \xi_{1}$, принадлежит $\mathbf{R}_{p}(X)$ и выполнено неравенство $\left(\mathbf{E}\left|\left\langle\xi_{1}, x^{*}\right\rangle\right|^{p}\right)^{1 / p} \leqslant c_{p}\left(\mathbf{E}\left\langle\xi_{1}, x^{*}\right\rangle^{2}\right)^{1 / 2} \partial \Omega A$ некоторых $p \geqslant 2$ и $c_{p}>0$. Тогда существует стохастический интеграл $\int_{0}^{1} \xi_{t} d W_{t}$. Более того, если $\left(x_{k}\right)_{k \in \mathbf{N}},\left(x_{k}^{*}\right)_{k \in \mathbf{N}}$ таковы, ито

$$
\begin{aligned}
& \left\langle R_{1} x_{k}^{*}, x_{j}^{*}\right\rangle=\delta_{k j}, \quad R_{1} x_{k}^{*}=x_{k}, \quad j, k=1,2, \ldots, \\
& R_{1} x^{*}=\sum_{k=1}^{\infty}\left\langle x^{*}, x_{k}\right\rangle x_{k}, \quad x^{*} \in X^{*},
\end{aligned}
$$

mo

$$
\xi_{t}=\sum_{k=1}^{\infty} x_{k}\left\langle\xi_{t}, x_{k}^{*}\right\rangle, \quad \int_{0}^{1} \xi_{t} d W_{t}=\sum_{k=1}^{\infty} x_{k} \int_{0}^{1}\left\langle\xi_{t}, x_{k}^{*}\right\rangle d W_{t}
$$

и оба ряда сходятся в $L_{p}(\Omega, X)$.

Д ок аз а т ель с т в о. Пусть $R_{1}=A A^{*}, A: H \rightarrow X, A^{*} x_{k}^{*}:=e_{k}$. Рассмотрим $\eta_{n}=\sum_{k=1}^{n} e_{k}\left\langle\xi_{1}, x_{k}^{*}\right\rangle$. Для любого $h \in H$

$$
\begin{aligned}
\left(\mathbf{E}\left|\left\langle\eta_{n}-\eta_{m}, h\right\rangle\right|^{p}\right)^{1 / p} & =\left(\mathbf{E}\left|\left\langle\xi_{1}, \sum_{k=n}^{m} h_{k} x_{k}^{*}\right\rangle\right|^{p}\right)^{1 / p} \\
& \leqslant c_{p}\left(\mathbf{E}\left\langle\xi_{1}, \sum_{k=n}^{m} h_{k} x_{k}^{*}\right\rangle^{2}\right)^{1 / 2}=c_{p}\left(\sum_{k=n}^{m} h_{k}^{2}\right)^{1 / 2} \rightarrow 0 .
\end{aligned}
$$


По лемме 6.5.2 из [2] $A \eta_{n}=\sum_{k=1}^{n} x_{k}\left\langle\xi_{1}, x_{k}^{*}\right\rangle$ сходится в $L_{p}(\Omega, X)$. Имеем

$$
\mathbf{E}\left(\sum_{k=1}^{n} x_{k}\left\langle\xi_{1}, x_{k}^{*}\right\rangle \mid \mathscr{F}_{t}\right)=\sum_{k=1}^{n} x_{k} \mathbf{E}\left(\left\langle\xi_{1}, x_{k}^{*}\right\rangle \mid \mathscr{F}_{t}\right)=\sum_{k=1}^{n} x_{k}\left\langle\xi_{t}, x_{k}^{*}\right\rangle
$$

и

$$
\begin{aligned}
& \left(\mathbf{E}\left\|\sum_{k=n}^{m} x_{k}\left\langle\xi_{t}, x_{k}^{*}\right\rangle\right\|^{p}\right)^{1 / p}=\left(\mathbf{E}\left\|\mathbf{E}\left(\sum_{k=n}^{m} x_{k}\left\langle\xi_{1}, x_{k}^{*}\right\rangle \mid \mathscr{F}_{t}\right)\right\|^{p}\right)^{1 / p} \\
& \quad \leqslant\left(\mathbf{E} \mathbf{E}\left(\left\|\sum_{k=n}^{m} x_{k}\left\langle\xi_{1}, x_{k}^{*}\right\rangle\right\|^{p} \mid \mathscr{F}_{t}\right)\right)^{1 / p}=\left(\mathbf{E}\left\|\sum_{k=n}^{m} x_{k}\left\langle\xi_{1}, x_{k}^{*}\right\rangle\right\|^{p}\right)^{1 / p} \rightarrow 0 .
\end{aligned}
$$

Следовательно, правая часть равенства $\xi_{t}=\sum_{k=1}^{\infty} x_{k}\left\langle\xi_{t}, x_{k}^{*}\right\rangle$ сходится в $L_{p}(\Omega, X)$.

Теперь рассмотрим $\zeta_{n}=\sum_{k=1}^{n} e_{k} \int_{0}^{1}\left\langle\xi_{t}, x_{k}^{*}\right\rangle d W_{t}$. Для любого $h \in H$

$$
\begin{aligned}
& \left(\mathbf{E}\left|\left\langle\zeta_{n}-\zeta_{m}, h\right\rangle\right|^{p}\right)^{1 / p}=\left(\mathbf{E}\left|\int_{0}^{1}\left\langle\xi_{t}, \sum_{k=n}^{m} h_{k} x_{k}^{*}\right\rangle d W_{t}\right|^{p}\right)^{1 / p} \\
& \quad \leqslant \lambda_{p}\left(\mathbf{E} \int_{0}^{1}\left|\left\langle\xi_{t}, \sum_{k=n}^{m} h_{k} x_{k}^{*}\right\rangle\right|^{p} d t\right)^{1 / p} \leqslant \lambda_{p}\left(\mathbf{E}\left|\left\langle\xi_{1}, \sum_{k=n}^{m} h_{k} x_{k}^{*}\right\rangle\right|^{p}\right)^{1 / p} \\
& \quad \leqslant \lambda_{p} c_{p}\left(\mathbf{E}\left\langle\xi_{1}, \sum_{k=n}^{m} h_{k} x_{k}^{*}\right\rangle^{2}\right)^{1 / 2}=\lambda_{p} c_{p} \sum_{k=n}^{m} h_{k}^{2} \rightarrow 0 .
\end{aligned}
$$

По лемме 6.5.2 из [2], $A \zeta_{n}=\sum_{k=1}^{n} x_{k} \int_{0}^{1}\left\langle\xi_{t}, x_{k}^{*}\right\rangle d W_{t}$ сходится в $L_{p}(\Omega, X)$. Тогда $\int_{0}^{1} \xi_{t} d W_{t}=\sum_{k=1}^{\infty} x_{k} \int_{0}^{1}\left\langle\xi_{t}, x_{k}^{*}\right\rangle d W_{t}$. Теорема доказана.

Теорема 5. Если неупреждающий ОСП $\left(\xi_{t}\right)_{t \in[0,1]}$ удовлетворяет условиям теоремь 2 или теоремь 3 , то для всех $m$ существуют стохастические интегральи

$$
\xi_{t}^{(1)}=\int_{0}^{t} \xi_{s} d W_{s}, \quad \ldots, \quad \xi_{t}^{(m)}=\int_{0}^{t} \xi_{s}^{(m-1)} d W_{s}
$$

и выполнено неравенство

$$
\left(\mathbf{E}\left\|\int_{0}^{1} \xi_{t}^{(m)} d W_{t}\right\|^{p}\right)^{1 / p} \leqslant \lambda_{p}^{m+1} c_{p} \Pi_{p}(R)
$$

где $R$ - ковариачионньй оператор случайного элемента $\xi_{1}^{(1)}$.

Д о к а з а т е ль с т в о. Пусть $\left(x_{k}\right)_{k \in \mathbf{N}}$ и $\left(x_{k}^{*}\right)_{k \in \mathbf{N}}$ такие, что

$$
\begin{gathered}
R x_{k}^{*}=x_{k}, \quad\left\langle R x_{k}^{*}, x_{j}^{*}\right\rangle=\delta_{k j}, \quad R=A A^{*}, \\
A^{*} x_{k}^{*}:=e_{k}, \quad R x^{*}=\sum_{k=1}^{\infty}\left\langle x_{k}, x^{*}\right\rangle x_{k} .
\end{gathered}
$$


Рассмотрим

$$
\eta_{n}=\sum_{k=1}^{n} e_{k} \int_{0}^{1}\left\langle\xi_{t}^{(1)}, x_{k}^{*}\right\rangle d W_{t}
$$

Для любого $h \in H$

$$
\begin{aligned}
\left(\mathbf{E} \mid\left\langle\eta_{m}-\right.\right. & \left.\left.\eta_{n}, h\right\rangle\left.\right|^{p}\right)^{1 / p}=\left(\mathbf{E}\left|\int_{0}^{1}\left\langle\xi_{t}^{(1)}, \sum_{k=n}^{m} h_{k} x_{k}^{*}\right\rangle d W_{t}\right|^{p}\right)^{1 / p} \\
\leqslant & \lambda_{p}\left(\mathbf{E} \int_{0}^{1}\left|\left\langle\xi_{t}^{(1)}, \sum_{k=n}^{m} h_{k} x_{k}^{*}\right\rangle\right|^{p} d t\right)^{1 / p} \\
& \times \lambda_{p}\left(\int_{0}^{1} \mathbf{E}\left|\int_{0}^{t}\left\langle\xi, \sum_{k=n}^{m} h_{k} x_{k}^{*}\right\rangle d W_{s}\right|^{p} d s\right)^{1 / p} \\
\leqslant & \lambda_{p}^{2}\left(\int_{0}^{1} \mathbf{E} \int_{0}^{t}\left|\left\langle\xi_{s}, \sum_{k=n}^{m} h_{k} x_{k}^{*}\right\rangle\right|^{p} d s d t\right)^{1 / p} \\
\leqslant & \lambda_{p}^{2}\left(\mathbf{E} \int_{0}^{1} \mid\left\langle\xi_{s}, \sum_{k=n}^{m} h_{k} x_{k}^{*}\right\rangle^{p} d s\right)^{1 / p} \\
\leqslant & \lambda_{p}^{2} c_{p}\left(\mathbf{E} \int_{0}^{1}\left\langle\xi_{s}, \sum_{k=n}^{m} h_{k} x_{k}^{*}\right\rangle^{2} d s\right)^{1 / 2}=\lambda_{p}^{2} c_{p} \sum_{k=n}^{m} h_{k}^{2} \rightarrow 0 .
\end{aligned}
$$

По лемме 6.5.2 из [2], $A \eta_{n}$ сходится в $L_{p}(\Omega, X)$ и

$$
\int_{0}^{1} \xi_{t}^{(1)} d W_{t}=\int_{0}^{1} \sum_{k=1}^{\infty} x_{k}\left(\int_{0}^{t}\left\langle\xi_{s}, x_{k}^{*}\right\rangle d W_{s}\right) d W_{t}=\sum_{k=1}^{\infty} x_{k} \int_{0}^{1}\left\langle\xi_{t}^{(1)}, x_{k}^{*}\right\rangle d W_{t} .
$$

Аналогично можно доказать, что

$$
\int_{0}^{1} \xi_{t}^{(m)} d W_{t}=\sum_{k=1}^{\infty} x_{k} \int_{0}^{1}\left\langle\xi_{t}^{(m-1)}, x_{k}^{*}\right\rangle d W_{t} .
$$

Оценим $\left(\mathbf{E}\left\|\int_{0}^{1} \xi_{t}^{(m)} d W_{t}\right\|^{p}\right)^{1 / p}$. Рассмотрим $\eta_{n}:=\sum_{k=1}^{n} e_{k} \int_{0}^{1}\left\langle\xi_{t}^{(m)}, x_{k}^{*}\right\rangle d W_{t}$. Так как $A: H \rightarrow X-p$-абсолютно суммирующий оператор, то по теореме А. Пичча (см. [2, теорема 2.2.2]) существует вероятностная мера $\nu$ на единичном шаре $H$ такая, что

$$
\begin{aligned}
\mathbf{E}\left\|A \eta_{n}(\omega)\right\|^{p} & \leqslant \Pi_{p}^{p}(A) \mathbf{E} \int_{\|h\| \leqslant 1}\left|\left\langle\eta_{n}(\omega), h\right\rangle\right|^{p} d \nu(h) \\
& =\Pi_{p}^{p}(A) \int_{\|h\| \leqslant 1} \mathbf{E}\left|\int_{0}^{1}\left\langle\xi_{t}^{(m)}(\omega), \sum_{k=1}^{n} h_{k} x_{k}^{*}\right\rangle d W_{t}\right|^{p} d \nu(h) \\
& \leqslant \Pi_{p}^{p}(A) \lambda_{p}^{(m+1) p} \int_{\|h\| \leqslant 1} \mathbf{E} \int_{0}^{1}\left|\left\langle\xi_{t}, \sum_{k=1}^{n} h_{k} x_{k}^{*}\right\rangle\right|^{p} d t d \nu \\
& \leqslant \Pi_{p}^{p}(A) \lambda_{p}^{p(m+1)} c_{p}^{p} \int_{\|h\| \leqslant 1}\left(\mathbf{E} \int_{0}^{1}\left\langle\xi_{t}, \sum_{k=1}^{n} h_{k} x_{k}^{*}\right\rangle^{2} d t\right)^{p / 2} d \nu(h)
\end{aligned}
$$




$$
\leqslant \Pi_{p}^{p}(A) \lambda_{p}^{(m+1) p} c_{p}^{p} \int_{\|h\| \leqslant 1}\left(\sum_{k=1}^{n} h_{k}^{2}\right)^{p / 2} d \nu(h) \leqslant \Pi_{p}^{p}(A) \lambda_{p}^{(m+1) p} c_{p}^{p}
$$

Следовательно,

$$
\left(\mathbf{E}\left\|\int_{0}^{1} \xi_{t}^{(m)} d W_{t}\right\|^{p}\right)^{1 / p}=\lim _{n \rightarrow \infty}\left(\mathbf{E}\left\|A \eta_{n}(\omega)\right\|^{p}\right)^{1 / p} \leqslant \Pi_{p}(A) \lambda_{p}^{(m+1)} c_{p} .
$$

Теорема доказана.

Пусть дан винеровский процесс $\left(B_{t}\right)_{t \in[0,1]}$ со значениами в $X$, т.е. $B_{0}=0$ п.н., $B$ - гауссовский процесс и имеет независимые приращения и $\mathbf{E}\left\langle B_{t}-B_{s}, x^{*}\right\rangle^{2}=(t-s)\left\langle R x^{*}, x^{*}\right\rangle$, где $R-$ гауссовская ковариация. Тогда справедливо следующее утверждение.

Следствие 3. Пусть положительно определенный и симметричный оператор $R$ принадлежит $\mathbf{R}_{p}$ для некоторого $p \geqslant 2$. Тогда существуют стохастические интеграль

$$
\xi_{t}^{(0)}=\int_{0}^{t} B_{s} d W_{s}, \quad \ldots, \quad \xi_{t}^{(m)}=\int_{0}^{t} \xi_{s}^{(m-1)} d W_{S}, \quad \ldots
$$

4. Стохастические дифференциальные уравнения. Будем рассматривать стохастические дифференциальные уравнения для ОСП.

О п р е д е л е н и е 5 . Неупреждающий ОСП $\left(T_{t}\right), t \in[0,1]$, называется обобщенным решением или сильным обобщенным решением стохастического дифференциального уравнения

$$
d T_{t}=a\left(t, T_{t}\right) d t+b\left(t, T_{t}\right) d W_{t}
$$

с $\mathscr{F}_{0}$-измеримым начальным условием $T_{0}=\mathscr{T}\left(\mathscr{T} x^{*}\right.$ является $\mathscr{F}_{0}$ измеримым для всех $\left.x^{*} \in X^{*}\right)$, где $a:[0,1] \times \mathscr{M}_{1} \rightarrow \mathscr{M}_{1}, b:[0,1] \times \mathscr{M}_{1} \rightarrow$ $\mathscr{M}_{1}$, если для любого $x^{*} \in X^{*}$ функции $a\left(t, T_{t}\right) x^{*}$ и $b\left(t, T_{t}\right) x^{*}$ являются измеримыми и неупреждающими,

$$
\mathbf{E} \int_{0}^{1}\left|a\left(t, T_{t}\right) x^{*}\right|^{2} d t+\mathbf{E} \int_{0}^{1}\left|b\left(t, T_{t}\right) x^{*}\right|^{2} d t<\infty
$$

И

$$
T_{t} x^{*}=\mathscr{T} x^{*}+\int_{0}^{t} a\left(s, T_{s}\right) x^{*} d s+\int b\left(s, T_{s}\right) x^{*} d W_{s} \quad \text { п.н. }
$$

О п р е д е л е н и е 6. Будем говорить, что стохастическое дифференциальное уравнение $(3)$ с $\mathscr{F}_{0}$-измеримым начальным условием $T_{0}=$ $\mathscr{T}$ имеет единственное сильное обобщенное решение, если для любых его двух сильных решений $\left(T_{t}\right)_{t \in[0,1]}$ и $\left(\bar{T}_{t}\right)_{t \in[0,1]}$

$$
\mathbf{P}\left(\omega: T_{t} x^{*}(\omega)=\bar{T}_{t} x^{*}(\omega) \text { для всех } t \in[0,1]\right)=1
$$

при любом фиксированном $x^{*} \in X^{*}$. 
Приведем теорему существования и единственности сильного обобщенного решения стохастического дифференциального уравнения.

Теорема 6. Пусть неупреждаюшие функиии а и $b$ удовлетворяют следуюшим условиям:

1) $\|a(t, T)\|_{\mathscr{M}_{1}}^{2}+\|b(t, T)\|_{\mathscr{M}_{1}}^{2} \leqslant K^{2}\left(1+\|T\|_{\mathscr{M}_{1}}^{2}\right)$,

2) $\|a(t, T)-a(t, L)\|_{\mathscr{M}_{1}}^{2}+\|b(t, T)-b(t, L)\|_{\mathscr{M}_{1}}^{2} \leqslant K^{2}\|T-L\|_{\mathscr{M}_{1}}^{2}$.

Тогда существует единственное сильное обобщенное решение $\left(T_{t}\right)_{t \in[0,1]}$ уравнения (3) с начальньлм условием $T_{0}=\mathscr{T}$. При этом $T:[0,1] \rightarrow \mathscr{M}_{1}$ есть непрерывное отображение.

Д о к а з а т е ль с т в о. Для любых $t \in[0,1]$ и $x^{*} \in X^{*}$ пусть $T_{t}^{0}=\mathscr{T}$ и

$$
\begin{aligned}
& T_{t}^{n} x^{*}=T_{t}^{0} x^{*}+\int_{0}^{t} a\left(s, T_{s}^{n-1}\right) x^{*} d s+\int_{0}^{t} b\left(s, T_{s}^{n-1}\right) x^{*} d W_{s}, \\
& \left\|T_{t}^{n+1}-T_{t}^{n}\right\|_{\mathscr{M}_{1}}^{2} \leqslant 2 \sup _{\left\|x^{*}\right\| \leqslant 1} \mathbf{E}\left(\int_{0}^{t}\left(a\left(s, T_{s}^{n}\right)-a\left(s, T_{s}^{n-1}\right)\right) x^{*} d s\right)^{2} \\
& \quad+2 \sup _{\left\|x^{*}\right\| \leqslant 1} \mathbf{E}\left(\int_{0}^{t}\left(b\left(s, T_{s}^{n}\right)-b\left(s, T_{s}^{n-1}\right)\right) x^{*} d W_{s}\right)^{2} \\
& \quad \leqslant 2 \int_{0}^{t}\left\|a\left(s, T_{s}^{n}\right)-a\left(s, T^{n-1}\right)_{s}\right\|_{\mathscr{M}_{1}}^{2} d s+2 \int_{0}^{t}\left\|b\left(s, T_{s}^{n}\right)-b\left(s, T_{s}^{n-1}\right)\right\|_{\mathscr{M}_{1}}^{2} d s \\
& \leqslant 2 K^{2} \int_{0}^{t}\left\|T_{s}^{n}-T_{s}^{n-1}\right\|_{\mathscr{M}_{1}}^{2} d s .
\end{aligned}
$$

По индукции получаем

$$
\begin{aligned}
\left\|T_{t}^{n+1}-T_{t}^{n}\right\|_{\mathscr{M}_{1}}^{2} & \leqslant\left(2 K^{2}\right)^{n-1} \int_{0}^{t} \frac{(t-s)^{n-1}}{(n-1) !}\left\|T_{s}^{1}-T_{s}^{0}\right\|_{\mathscr{M}_{1}}^{2} d s \\
\left\|T_{s}^{1}-T_{s}^{0}\right\|_{\mathscr{M}_{1}}^{2} & \leqslant 2\left\|\int_{0}^{t} a\left(s, T_{s}^{0}\right) d s\right\|_{\mathscr{M}_{1}}^{2}+2\left\|\int_{0}^{t} b\left(s, T_{s}^{0}\right) d W_{s}\right\|_{\mathscr{M}_{1}}^{2} \\
& \leqslant 2 K^{2}\left(1+\left\|T_{0}\right\|_{\mathscr{M}_{1}}^{2}\right) .
\end{aligned}
$$

Следовательно, $\left\|T_{t}^{n+1}-T_{t}^{n}\right\|_{\mathscr{M}_{1}}^{2} \leqslant p C^{n} / n$ ! для некоторых положительных $p$ и $C$.

Для любого фиксированного $x^{*} \in X^{*}$

$$
\begin{aligned}
\mathbf{E} & \sup _{0 \leqslant t \leqslant 1}\left|\left(T_{t}^{n+1}-T_{t}^{n}\right) x^{*}\right|^{2} \leqslant 2 \mathbf{E} \sup _{0 \leqslant t \leqslant 1} \int_{0}^{t}\left(\left(a\left(s, T_{s}^{n}\right)-a\left(s, T_{s}^{n-1}\right)\right) x^{*}\right)^{2} d s \\
& +2 \mathbf{E} \sup _{0 \leqslant t \leqslant 1}\left|\int_{0}^{t}\left(b\left(s, T_{s}^{n}\right)-b\left(s, T_{s}^{n-1}\right)\right) x^{*} d W_{s}\right|^{2} \\
& \leqslant 2 \int_{0}^{1}\left\|a\left(s, T_{s}^{n}\right)-a\left(s, T_{s}^{n-1}\right)\right\|_{\mathscr{M}_{1}}^{2} d s+8 \int_{0}^{1}\left\|b\left(s, T_{s}^{n}\right)-b\left(s, T_{s}^{n-1}\right)\right\|_{\mathscr{M}_{1}}^{2} d s \\
& \leqslant \frac{10 p C^{n-1}}{(n-1) !}
\end{aligned}
$$


Тогда мы имеем

$$
\begin{aligned}
\sum_{n=1}^{\infty} \mathbf{P}\left(\sup _{0 \leqslant t \leqslant 1}\left|\left(T_{t}^{n+1}-T_{t}^{n}\right) x^{*}\right|>\frac{1}{n^{2}}\right) & \leqslant \sum_{n=1}^{\infty} n^{4} \mathbf{E}\left(\sup _{0 \leqslant t \leqslant 1}\left|\left(T_{t}^{n+1}-T_{t}^{n}\right) x^{*}\right|^{2}\right) \\
& \leqslant 10 p \sum_{n=1}^{\infty} \frac{n^{4} C^{n-1}}{(n-1) !} .
\end{aligned}
$$

По лемме Бореля-Кантелли ряд

$$
T_{t}^{0} x^{*}(\omega)+\sum_{m=1}^{\infty}\left(T_{t}^{n}(\omega)-T_{t}^{n-1}(\omega)\right) x^{*}
$$

сходится равномерно по $t$ (P-п.н.) к непрерывному случайному процессу, который обозначим через $T_{t} x^{*}, x^{*} \in X^{*}$. Следовательно, получаем ОСП $T_{t}: X^{*} \rightarrow L_{2}(\Omega, \mathscr{B}, \mathbf{P})$. Из уравнения (2) имеем

$$
T_{t} x^{*}=\mathscr{T} x^{*}+\int_{0}^{t} a\left(s, T_{s}\right) x^{*} d s+\int b\left(s, T_{s}\right) x^{*} d W_{s} \quad \text { п.н. }
$$

Построенный таким образом ОСП $\left(T_{t}\right), t \in[0,1]$, является решением уравнения (1).

Можно оценить значение $\left\|T_{t}\right\|_{\mathscr{M}_{1}}, t \in[0,1]$ :

$$
\begin{aligned}
\left\|T_{t}^{n}\right\|_{\mathscr{M}_{1}}^{2} & \leqslant 3\left(\|\mathscr{T}\|_{\mathscr{M}_{1}}^{2}+3 K \int_{0}^{t}\left(1+\left\|T_{s}^{(n-1)}\right\|_{\mathscr{M}_{1}}^{2}\right) d s\right) \\
& \leqslant 3\left(\|\mathscr{T}\|_{\mathscr{M}_{1}}^{2}+K^{2}\right)+3 K^{2} \int_{0}^{t}\left\|T_{s}^{(n-1)}\right\|_{\mathscr{M}_{1}}^{2} d s \\
& \leqslant 3\left(K^{2}+\|\mathscr{T}\|_{\mathscr{M}_{1}}^{2}\right)\left(1+3 K^{2}\right)+9 K^{4} \int_{0}^{t} \int_{0}^{s}\left\|T_{u}^{(n-2)}\right\|_{\mathscr{M}_{1}}^{2} d u d s
\end{aligned}
$$

Отсюда вытекает неравенство

$$
\left\|T_{t}^{n}\right\|_{\mathscr{M}_{1}}^{2} \leqslant 3\left(K^{2}+\|\mathscr{T}\|_{\mathscr{M}_{1}}^{2}\right) \exp \left(3 K^{2}\right)
$$

которое справедливо для любого $n$. Следовательно, оно справедливо и для $T_{t}, t \in[0,1]$.

Докажем непрерывность $T_{t}^{n}$ и $T_{t}$. Для $T_{t}^{n}$ имеем:

$$
\begin{aligned}
\left\|T_{t}^{n}-T_{s}^{n}\right\|_{\mathscr{M}_{1}}^{2} & \leqslant 2 \int_{s}^{t}\left(\left\|a\left(u, T_{u}^{(n-1)}\right)\right\|_{\mathscr{M}_{1}}^{2}+\left\|b\left(u, T_{u}^{(n-1)}\right)\right\|_{\mathscr{M}_{1}}^{2}\right) d u \\
& \leqslant 2 K^{2} \int_{s}^{t}\left(1+\left\|T_{u}^{(n-1)}\right\|_{\mathscr{M}_{1}}^{2}\right) d u \\
& \leqslant(t-s) \cdot 6 K^{2}\left(K^{2}+\|\mathscr{T}\|_{\mathscr{M}_{1}}^{2} \exp \left(3 K^{2}\right)\right) \rightarrow 0
\end{aligned}
$$

при $s \rightarrow t$. Отсюда получаем и непрерывность $T_{t}$. 
Единственность. Пусть $\left(T_{t}\right), t \in[0,1]$, и $\left(\bar{T}_{t}\right), t \in[0,1],-$ два решения с $T_{0}=\bar{T}_{0}=\mathscr{T}$. Тогда

$$
\begin{aligned}
\left\|T_{t}-\bar{T}_{t}\right\|_{\mathscr{M}_{1}}^{2} \leqslant & 2 t \sup _{\left\|x^{*}\right\| \leqslant 1} \mathbf{E} \int_{0}^{t}\left(a\left(s, T_{s}\right)-a\left(s, \bar{T}_{s}\right)\right)\left(x^{*}\right)^{2} d s \\
& +2 \sup _{\left\|x^{*}\right\| \leqslant 1} \mathbf{E} \int_{0}^{t}\left(b\left(s, T_{s}\right)-b\left(s, \bar{T}_{s}\right)\right)\left(x^{*}\right)^{2} d s \\
\leqslant & 2 t \int_{0}^{t}\left\|a\left(s, T_{s}\right)-a\left(s, \bar{T}_{s}\right)\right\|_{\mathscr{M}_{1}}^{2} d s \\
& +2 \int_{0}^{t}\left\|b\left(s, T_{s}\right)-b\left(s, \bar{T}_{s}\right)\right\|_{\mathscr{M}_{1}}^{2} d s \leqslant L \int_{0}^{t}\left\|T_{s}-\bar{T}_{s}\right\|_{\mathscr{M}_{1}}^{2} d s
\end{aligned}
$$

где $L=2 t K^{2}+2 K^{2}$. Имеем

$$
\left\|T_{t}-\bar{T}_{t}\right\|_{\mathscr{M}_{1}}^{2} \leqslant L \int_{0}^{t}\left\|T_{s}-\bar{T}_{s}\right\|_{\mathscr{M}_{1}}^{2} d s
$$

Следовательно, $\left\|T_{t}-\bar{T}_{t}\right\|_{\mathscr{M}_{1}}^{2}=0$, тогда для любого $t \in[0,1]$ и $x^{*} \in X^{*}$ выполнено равенство $\mathbf{P}\left(T_{t} x^{*}=\bar{T}_{t} x^{*}\right)=1$. Из непрерывности реализаций этих процессов получаем

$$
\mathbf{P}\left(\omega: T_{t} x^{*}(\omega)=\bar{T}_{t} x^{*}(\omega) \text { для всех } t \in[0,1]\right)=1,
$$

что и завершает доказательство теоремы 6.

Рассмотрим стохастическое дифференциальное уравнение в сепарабельном банаховом пространстве $X$ :

$$
d \xi_{t}=a\left(t, \xi_{t}\right) d t+b\left(t, \xi_{t}\right) d W_{t}
$$

где $a:[0,1] \times X \rightarrow X, b:[0,1] \times X \rightarrow X-$ такие $\mathscr{B}[0,1] \times \mathscr{B}(X)$-измеримые функции, что

1) $\|a(t, \xi)\|_{\mathscr{M}_{1}}^{2}\|b(t, \xi)\|_{\mathscr{M}_{1}}^{2} \leqslant K^{2}\left(1+\|\xi\|_{\mathscr{M}_{1}}\right)^{2}$

2) $\|a(t, \xi)-a(t, \eta)\|_{\mathscr{M}_{1}}^{2}+\|b(t, \xi)-b(t, \eta)\|_{\mathscr{M}_{1}}^{2} \leqslant K^{2}\|\xi-\eta\|_{\mathscr{M}_{1}}^{2}$,

где $\xi, \eta$ - случайные элементы со значением в $X$.

Если условия 1) и 2) выполнены, то можно продолжить функции $a$ и $b$ на $\overline{\mathscr{M}}_{2} \subseteq \mathscr{M}_{1}$ : пусть $T \in \overline{\mathscr{M}}_{2}$, тогда существует последовательность $\left(\xi_{n}\right)_{n \in \mathbf{N}} \in \mathscr{M}_{2}$ такая, что $\left\|\xi_{n}-T\right\|_{\mathscr{M}_{1}} \rightarrow 0$. Поскольку $\left\|a\left(t, \xi_{n}\right)-a\left(t, \xi_{m}\right)\right\|_{\mathscr{M}_{1}}^{2} \leqslant K^{2}\left\|\xi_{n}-\xi_{m}\right\|_{\mathscr{M}_{1}}^{2} \rightarrow 0$, то $a(t, T):=\lim _{n \rightarrow \infty} a\left(t, \xi_{n}\right)$. Аналогично, $b(t, T):=\lim _{n \rightarrow \infty} b\left(t, \xi_{n}\right)$. Следовательно, для любого $T \in$ $\overline{\mathscr{M}}_{2}, a(t, T)$ и $b(t, T)$ принадлежат $\overline{\mathscr{M}}_{2}$.

Для любых $T$ и $L$ из $\overline{\mathscr{M}}_{2}$ справедливы также неравенства

$\left.1^{\prime}\right)\|a(t, T)\|_{\mathscr{M}_{1}}^{2}+\|b(t, T)\|_{\mathscr{M}_{1}}^{2} \leqslant K^{2}\left(1+\|T\|_{\mathscr{M}_{1}}^{2}\right)$,

$\left.2^{\prime}\right)\|a(t, T)-a(t, L)\|_{\mathscr{M}_{1}}^{2}+\|b(t, T)-b(t, L)\|_{\mathscr{M}_{1}}^{2} \leqslant K^{2}\|T-L\|_{\mathscr{M}_{1}}^{2}$. 
Следовательно, из уравнения (5) получаем стохастическое дифференциальное уравнение для ОСП

$$
d T_{t}=a\left(t, T_{t}\right) d t+b\left(t, T_{t}\right) d W_{t}
$$

с $\mathscr{F}_{0}$-измеримым начальным условием $T_{0} x^{*}=\left\langle\xi_{0}, x^{*}\right\rangle$, где измеримые неупреждающие функции $a$ и $b$ удовлетворяют условиям $\left.1^{\prime}\right)$ и $2^{\prime}$ ). Поэтому справедлива следующая теорема.

Теорема 7. Пусть измеримье неупреждаюшие функции а и $b$ удовлетворяют условиям 1), 2) и для любого случайного элемента $\xi \in \mathscr{M}_{2}$ отображения $a(\cdot, \xi)$ и $b(\cdot, \xi)$ из $[0,1]$ в $\mathscr{M}_{1}$ непрерьввнь. Тогда стохастическое дифференциальное уравнение (5) имеет единственное обобшенное решение $T_{t}$ с начальныл условием $T_{0} x^{*}=\left\langle\xi_{0}, x^{*}\right\rangle$, где $\xi_{0} \in \mathscr{M}_{2}$ есть $\mathscr{F}_{0}$-измеримый случайный элемент. Решение $T(t)$ таково, что $T(t) \in \overline{\mathscr{M}}_{2}$.

Д о к а з а т е л ь с т в о. Применяя теорему 6 , достаточно доказать, что $\int_{0}^{1} a\left(t, T_{t}^{n}\right) d t$ и $\int_{0}^{1} b\left(t, T_{t}^{n}\right) d W_{t}$ принадлежат $\overline{\mathscr{M}_{2}}$, где $T_{t}^{0} x^{*}=$ $\left\langle\xi_{0}, x^{*}\right\rangle$ и для любого $x^{*} \in X^{*}$

$$
T_{t}^{n} x^{*}=T_{t}^{0} x^{*}+\int_{0}^{t} a\left(s, T_{s}^{n-1}\right) x^{*} d s+\int_{0}^{t} b\left(s, T_{s}^{n-1}\right) x^{*} d W_{s} .
$$

Мы уже показали, что если $T \in \mathscr{\mathscr { M }}_{2}$, то $a(t, T)$ и $b(t, T)$ тоже принадлежат $\overline{\mathscr{M}}_{2}$. Из непрерывности $a(t, \xi)$ и $b(t, \xi)$ для любого $\xi \in \mathscr{M}_{2}$ следует непрерывность $a(t, T)$ и $b(t, T)$ для всех $T \in \overline{\mathscr{M}}_{2}$. Для любого $n \in \mathbf{N}$ функции $a\left(t, T_{t}^{n}\right)$ и $b\left(t, T_{t}^{n}\right)$ непрерывны, а значит, являются сепарабельнозначными функциями в $\overline{\mathscr{M}}_{2}$.

Так как $\left\|a\left(t, T_{t}^{n}\right)\right\|_{\mathscr{M}_{1}}^{2}+\left\|b\left(t, T_{t}^{n}\right)\right\|_{\mathscr{M}_{1}}^{2} \leqslant K^{2}\left(1+\left\|T_{t}^{n}\right\|_{\mathscr{M}_{1}}^{2}\right) \leqslant C$ и $a(\cdot, T)$, $b(\cdot, T)$ - сепарабельнозначные функции, то $\int_{0}^{1} a\left(t, T_{t}^{n}\right) d t \in \overline{\mathscr{M}_{2}}$ и, по теореме $2, \int_{0}^{1} b\left(t, T_{t}^{n}\right) d W_{t} \in \overline{\mathscr{M}}_{2}$. Следовательно, можно применить теорему 6 , что завершает доказательство теоремы.

3 а м е ч а н и е. Непрерывность $a(\cdot, \xi)$ и $b(\cdot, \xi)$ нужна для сепарабельности области значения этих функций в $\mathscr{M}_{2}$, что является более слабым требованием, чем непрерывность.

Рассмотрим сейчас линейное стохастическое дифференциальное уравнение в произвольном сепарабельном банаховом пространстве:

$$
d \xi_{t}=A(t) \xi_{t} d t+B(t) \xi_{t} d W_{t}
$$

где $A:[0,1] \rightarrow L(X, X)$ и $B:[0,1] \rightarrow L(X, X)$ непрерывны. Тогда

$$
\max \left(\sup _{t \in[0,1]}\|A(t)\|, \sup _{t \in[0,1]}\|B(t)\|\right) \leqslant M
$$


для некоторого $M>0$. Имеем

$$
\begin{aligned}
\|A(t) \xi-A(t) \eta\|_{\mathscr{M}_{1}}^{2} & =\sup _{\left\|x^{*}\right\| \leqslant 1} \mathbf{E}\left\langle A(t)(\xi-\eta), x^{*}\right\rangle^{2} \\
& =\sup _{\left\|x^{*}\right\| \leqslant 1} \mathbf{E}\left\langle\xi-\eta, A^{*}(t) x^{*}\right\rangle^{2} \leqslant \sup _{\left\|x^{*}\right\| \leqslant M} \mathbf{E}\left\langle\xi-\eta, x^{*}\right\rangle^{2} \\
& =M^{2} \sup _{\left\|x^{*}\right\| \leqslant 1} \mathbf{E}\left\langle\xi-\eta, x^{*}\right\rangle^{2}=M^{2}\|\xi-\eta\|_{\mathscr{M}_{1}}^{2} .
\end{aligned}
$$

Аналогично можно показать, что $A$ и $B$ удовлетворяют условиям 1$)$ и 2 ) теоремы 6. Легко доказать, что отображения $A(t) \xi$ и $B(t) \xi$ из $[0,1]$ в $\mathscr{M}_{1}$ непрерывны. Таким образом, из теоремы 7 получаем следующий результат.

Теорема 8. Если коэффициенть $A$ и В непрерыьвны, то линейное стохастическое дифференциальное уравнение (4) имеет единственное обобщенное решение $T_{t}$ с начальньлм условием $T_{0} x^{*}=\left\langle\xi_{0}, x^{*}\right\rangle$, где $\xi_{0}: \Omega \rightarrow X$ является $\mathscr{F}_{0}$-измеримьл случайньл элементом. При этом $T(t) \in \overline{\mathscr{M}}_{2}$.

Мы получили ОСП $T(t)$ как обобщенное решение стохастического дифференциального уравнения в сепарабельном банаховом пространстве при не очень жестких условиях. Мы показали также, что это решение принадлежит $T \overline{\mathscr{M}}_{2}$, значит, по предложению 2 , существуют $m:[0,1] \rightarrow X$ такое, что $\mathbf{E} T_{t} x^{*}=\left\langle m(t), x^{*}\right\rangle$, и $R_{t}: X^{*} \rightarrow X$ такое, что $\left\langle R_{t} x^{*}, y^{*}\right\rangle=\mathbf{E} T_{t} x^{*} T_{t} y^{*}$. В следующей теореме предложены дополнительные достаточные условия, при которых существует уже сильное решение стохастического дифференциального уравнения.

Теорема 9. Пусть дано обобщенное решение $\left(T_{t}\right), t \in[0,1]$, из теоремь 7 или теоремь 8. Обозначим $L_{t} x^{*}:=T_{t} x^{*}-\left\langle m(t), x^{*}\right\rangle$. Если для некоторого фиксированного $p \geqslant 2$ и любого $x^{*} \in X^{*}$ выполнено неравенство $\left(\mathbf{E}\left(L_{t} x^{*}\right)^{p}\right)^{1 / p} \leqslant\left(\mathbf{E}\left(L_{t} x^{*}\right)^{2}\right)^{1 / 2}$ u $R_{t} \in \mathbf{R}_{p}(X)$, где $R_{t}-$ ковариачионный оператор ОСЭ $T_{t}$, то существует случайный прочесс $\left(\xi_{t}\right)$, $t \in[0,1]$, такой, что

$$
\xi_{t}=\xi_{0}+\int_{0}^{t} a\left(s, \xi_{s}\right) d s+\int_{0}^{t} b\left(s, \xi_{s}\right) d W_{s} .
$$

Д о к а з а т е л ь с т в о. Для произвольного $t \in[0,1]$ покажем, что существует случайный элемент $\xi_{t}$ такой, что $\left\langle\xi_{t}, x^{*}\right\rangle=T_{t} x^{*}$ для любого $x^{*} \in X^{*}$. Обозначим $H_{0}:=\left\{L_{t} x^{*}, x^{*} \in X^{*}\right\}$. Пусть $H-$ его замыкание в $L_{2}(\Omega, \mathscr{B}, \mathbf{P})$. Из условия теоремы следует, что $H \subset L_{p}(\Omega, \mathscr{B}, \mathbf{P})$. Профакторизуем оператор $R_{t}$ через гильбертово пространство $H: R_{t}=L_{t} L_{t}^{*}$. Так как $R_{t} \in \mathbf{R}_{p}(X)$, то $L_{t}^{*}: H \rightarrow X$ есть $p$-абсолютно суммирующий оператор. Пусть $A: H \rightarrow L_{p}(\Omega, \mathscr{B}, \mathbf{P})$ - естественное вложение. По теореме 
Л. Шварца (см. [11]), существует случайный элемент $\eta_{t} \in L_{p}(\Omega, X)$ такой, что $A L_{t} x^{*}=\left\langle\eta_{t}, x^{*}\right\rangle$ для любого $x^{*} \in X^{*}$. Тогда случайный процесс $\xi_{t}=\eta_{t}+m_{t}$ будет искомым решением. Теорема доказана.

Пусть сейчас коэффициенты стохастического дифференциального уравнения (5) таковы, что для некоторых $K>0$, положительно определенного, симметричного линейного оператора $R: X^{*} \rightarrow X$ и ограниченных линейных операторов $A_{i}: X \rightarrow X, i=1,2, \ldots$, таких, что $\sum_{i=1}^{\infty}\left\|A_{i}\right\|^{2}<\infty$, справедливы утверждения
a) $\left\langle a(t, x), x^{*}\right\rangle^{2}+\left\langle b(t, x), x^{*}\right\rangle^{2} \leqslant K^{2}\left(\left\langle R x^{*}, x^{*}\right\rangle+\sum_{i=1}^{\infty}\left\langle A_{i} x, x^{*}\right\rangle^{2}\right)$,
b) $\left\langle a(t, x)-a(t, y), x^{*}\right\rangle^{2}+\left\langle b(t, x)-b(t, y), x^{*}\right\rangle^{2} \leqslant K^{2} \sum_{i=1}^{\infty}\left\langle A_{i}(x-y), x^{*}\right\rangle^{2}$

для любых $t \in[0,1], x, y \in X$ и $x^{*} \in X^{*}$.

Тогда легко видеть, что коэффициенты $a$ и $b$ удовлетворяют условиям 1) и 2) теоремы 7 . Действительно,

$$
\begin{aligned}
\|a(t, \xi)\|_{\mathscr{M}_{1}}^{2} & =\sup _{x^{*} \in X^{*}} \mathbf{E}\left\langle a(T, \xi), x^{*}\right\rangle^{2} \\
& \leqslant \sup _{\left\|x^{*}\right\| \leqslant 1} K^{2}\left(\left\langle R x^{*}, x^{*}\right\rangle+\mathbf{E} \sum_{i=1}^{\infty}\left\langle A_{i} \xi, x^{*}\right\rangle^{2}\right) \\
& \leqslant K^{2}\left(\|R\|^{2}+\sup _{\left\|x^{*}\right\| \leqslant 1} \sum_{i=1}^{\infty}\left\langle\xi, \frac{A_{i}^{*}}{\left\|A_{i}^{*}\right\|} x^{*}\right\rangle^{2}\left\|A_{i}\right\|^{2}\right) \\
& \leqslant K^{2}\left(\|R\|+\|\xi\|_{\mathscr{M}_{1}}^{2} \sum_{i=1}^{\infty}\left\|A_{i}\right\|^{2}\right) \leqslant K_{1}^{2}\left(1+\|\xi\|_{\mathscr{M}_{1}}^{2}\right) .
\end{aligned}
$$

Аналогично получается второе неравенство. Следовательно, по теореме 7 , соответствующее стохастическое дифференциальное уравнение имеет единственное сильное обобщенное решение, принадлежащее $\overline{\mathscr{M}}_{2}$.

Следующая теорема (которая легко следует из теоремы 9) дает достаточное условие существования сильного решения стохастического дифференциального уравнения в произвольном сепарабельном банаховом пространстве (см. [5, теорема 2]).

Теорема 10. Пусть $\left(T_{t}\right)_{t \in[0,1]}$ - обобщенное решение стохастического дифференциального уравнения (5) с начальньм условием $T_{0} x^{*}=$ $\left\langle\xi_{0}, x^{*}\right\rangle$, коэффициентьг а и $b$ которого удовлетворяют условиям а), b), и существует число $p \geqslant 2$ такое, ито $\left(\mathbf{E}\left|T_{t} x^{*}-\mathbf{E} T_{t} x^{*}\right|^{p}\right)^{1 / p} \leqslant$ $\left(\mathbf{E}\left|T_{t} x^{*}-\mathbf{E} T_{t} x^{*}\right|^{2}\right)^{1 / 2}$ для всех $x^{*} \in X^{*}$. Ecли $R$ и $R_{0}:=R_{\xi_{0}}$ принадлежат $\mathbf{R}_{p}(X)$ и $\sum_{i=1}^{\infty}\left\|A_{i}\right\|<\infty$, то существует единственное сильное решение $\left(\xi_{t}\right)_{t \in[0,1]}$ стохастического дифференчиального уравнения (5) и $\mathbf{E}\left\|\xi_{t}\right\|^{p}<\infty$. 
Д о к а 3 а т е л ь с т в о. По теореме 9 и леммам 1 и 2 , достаточно оценить ковариационный оператор $R_{t}$ ОСЭ $T_{t}, t \in[0,1]$, ковариационным оператором $\bar{R}$ из класса $\mathbf{R}_{p}(X)$. Заметим, что линейные ограниченные операторы $A_{i}: X \rightarrow X, i \in \mathbf{N}$, можно продолжить на $\overline{\mathscr{M}}_{2}$. Имеем: $\left\langle R_{t} x^{*}, x^{*}\right\rangle=\lim _{n \rightarrow \infty} \mathbf{E}\left(T_{t}^{n} x^{*}\right)^{2}$ и

$$
\begin{aligned}
\mathbf{E}\left(T_{t}^{n} x^{*}\right)^{2} \leqslant & 3 \mathbf{E}\left\langle\xi_{0}, x^{*}\right\rangle^{2}+3 E \int_{0}^{t}\left(a\left(s, T_{s}^{(n-1)}\right) x^{*}\right)^{2} d s \\
& +3 \mathbf{E} \int_{0}^{t}\left(b\left(s, T_{s}^{(n-1)}\right) x^{*}\right)^{2} d s \\
\leqslant & 3\left\langle R_{0} x^{*}, x^{*}\right\rangle+3 K^{2} t\left\langle R x^{*}, x^{*}\right\rangle+3 K^{2} \int_{0}^{t} \sum_{i=1}^{\infty} \mathbf{E}\left(T_{s}^{n-1} A_{i}^{*} x^{*}\right)^{2} d s, \\
\mathbf{E}\left(T_{t}^{1} x^{*}\right)^{2} \leqslant & 3\left\langle R_{0} x^{*}, x^{*}\right\rangle+3 t K^{2}\left\langle R x^{*}, x^{*}\right\rangle+3 t K^{2} \sum_{i=1}^{\infty}\left\langle A_{i} R_{0} A_{i}^{*} x^{*}, x^{*}\right\rangle \\
\mathbf{E}\left(T_{t}^{2} x^{*}\right)^{2} \leqslant & 3\left\langle R_{0} x^{*}, x^{*}\right\rangle+3 t K^{2}\left\langle R x^{*}, x^{*}\right\rangle+3 K^{2} \int_{0}^{t} \sum_{i=1}^{\infty} \mathbf{E}\left(T_{s}^{1} A_{i}^{*} x^{*}\right)^{2} d s \\
\leqslant & 3\left\langle R_{0} x^{*}, x^{*}\right\rangle+3 t K^{2}\left\langle R x^{*}, x^{*}\right\rangle+3^{2} K^{2} t \sum_{i=1}^{\infty}\left\langle A_{i} R_{0} A_{i}^{*} x^{*}, x^{*}\right\rangle \\
& +\frac{3^{2} K^{4} t^{2}}{2} \sum_{i=1}^{\infty}\left\langle A_{i} R A_{i}^{*} x^{*}, x^{*}\right\rangle \\
& +\frac{3^{2} K^{4} t^{2}}{2} \sum_{i=1}^{\infty} \sum_{j=1}^{\infty}\left\langle A_{i} A_{j} R_{0} A_{j}^{*} A_{i}^{*} x^{*}, x^{*}\right\rangle .
\end{aligned}
$$

Соответственно,

$$
\begin{aligned}
\mathbf{E}\left(T_{t}^{n} x^{*}\right)^{2} \leqslant & 3\left\langle R_{0} x^{*}, x^{*}\right\rangle \\
& +3 \sum_{m=1}^{n} \frac{\left(3 K^{2} t\right)^{m}}{m !} \sum_{i_{1}=1}^{\infty} \cdots \sum_{i_{m}=1}^{\infty}\left\langle A_{i_{m}} \cdots A_{i_{1}} R_{0} A_{i_{1}}^{*} \cdots A_{i_{m}}^{*} x^{*}, x^{*}\right\rangle \\
& +3 t K^{2}\left\langle R x^{*}, x^{*}\right\rangle \\
& +\sum_{m=1}^{n-1} \frac{\left(3 k^{2} t\right)^{m+1}}{(m+1) !} \sum_{i_{1}=1}^{\infty} \cdots \sum_{i_{m}=1}^{\infty}\left\langle A_{i_{m}} \cdots A_{i_{1}} R A_{i_{1}}^{*} \cdots A_{i_{m}}^{*} x^{*}, x^{*}\right\rangle .
\end{aligned}
$$

Так как

$$
\begin{aligned}
& \Pi_{p}\left(\sum_{i_{1}=1}^{\infty} \cdots \sum_{i_{m}=1}^{\infty} A_{i_{m}} \cdots A_{i_{1}} R A_{i_{1}}^{*} \cdots A_{i_{m}}^{*}\right) \\
& \quad \leqslant \sum_{i_{1}=1}^{\infty} \cdots \sum_{i_{m}=1}^{\infty}\left\|A_{i_{1}}\right\| \cdots\left\|A_{i_{m}}\right\| \Pi_{p}(R)=\left(\sum_{i=1}^{\infty}\left\|A_{i}\right\|\right)^{m} \Pi_{p}(R),
\end{aligned}
$$

имеем

$$
\Pi_{p}\left(R_{t}\right) \leqslant C \sum_{m=0}^{\infty} \frac{\left(3 K^{2} t\right)^{m}}{m !}\left(\sum_{i=1}^{\infty}\left\|A_{i}\right\|\right)^{m} \Pi_{p}\left(R_{0}\right)
$$




$$
+C \sum_{m=0}^{\infty} \frac{\left(3 K^{2} t\right)^{m}}{m !}\left(\sum_{i=1}^{\infty}\left\|A_{i}\right\|\right)^{m} \Pi_{p}(R)<\infty
$$

Таким образом, $R_{t} \in \mathbf{R}_{p}(X)$, т.е. существует $\left(\xi_{t}\right)_{t \in[0,1]}$ такой, что $\left\langle\xi_{t}, x^{*}\right\rangle=T_{t} x^{*}, x^{*} \in X^{*}$. Теорема доказана.

\section{СПИСОК ЛИТЕРАТУРЫ}

1. Brzeźniak Z., van Neerven J.M.A.M., Veraar M. C., Weis L. Itô's formula in UMD Banach spaces and regularity of solutions of the Zakai equation. - J. Differential Equations, 2008, v. 245, p. 30-58.

2. Вахания Н.Н., Тариеладзе В.И., Чобанян С.А. Вероятностные распределения в банаховых пространствах. М.: Наука, 1985, 386 с.

3. Хилле Э., Филлипс Р. Функциональный анализ. М.: ИЛ, 1962, 829 с.

4. Mamporia B.I. Wiener processes and stochastic integrals on a Banach space. Probab. Math. Statist., 1986, v. 7, № 1, p. 59-75.

5. Mamporia B. I. On the existence and uniqueness of a solution to a stochastic differential equation in a Banach space. - Georgian Math. J., 2004, v. 11, № 3, p. 515-526.

6. McConnell T. R. Decoupling and stochastic integration in UMD Banach spaces. Probab. Math. Statist., 1989, v. 10, № 2, p. 283-295.

7. van Neerven J. M. A. M., Weis L. Stochastic integration of operator-valued functions with respect to Banach space-valued Brownian motion. - Potential Anal., 2008, v. 29, № 1 , p. $65-88$.

8. Pisier G. Martingales with values in uniformly convex spaces. - Israel J. Math., 1975 , v. 20, p. 326-350.

9. Rosiński J., Suchanecki Z. On the space of vector-valued functions integrable with respect to the white noise. - Colloq. Math., 1980, v. 43, № 1, p. 183-201.

10. Розовский Б. Л. Эволюционные стохастические системы. М.: Наука, 1983, 208 с.

11. Schwartz L. Application p-radonifiantes. - Séminaire L. Schwartz 1969/70, Exp. 11, 12.

12. Yang W. Zh., Tsai T. M. Itô's integration on 2-smooth Banach space. - Chinese J. Math., 1983, v. 11, p. 193-206. 